\title{
Germanica
}

\section{Les Philosophes au Pays des Spectres}

Die Philosophen im Geisterreich

\section{Louis Vax}

\section{(2) OpenEdition}

\section{Journals}

Édition électronique

URL : http://journals.openedition.org/germanica/2822

DOI : 10.4000/germanica.2822

ISSN : 2107-0784

\section{Éditeur}

Université de Lille

\section{Édition imprimée}

Date de publication : 30 juin 1988

Pagination : 51 à 116

ISSN : 0984-2632

\section{Référence électronique}

Louis Vax, "Les Philosophes au Pays des Spectres », Germanica [En ligne], $3 \mid$ 1988, mis en ligne le 17 juillet 2015, consulté le 06 octobre 2020. URL : http://journals.openedition.org/germanica/2822 ; DOI : https://doi.org/10.4000/germanica.2822

Ce document a été généré automatiquement le 6 octobre 2020.

(c) Tous droits réservés 


\title{
Les Philosophes au Pays des Spectres
}

\author{
Die Philosophen im Geisterreich
}

Louis Vax

I

... wo Gespenster Platz genommen

Ist auch der Philosoph willkommen

Faust II, 7643-4.

1 Cependant plus d'un a décliné l'invitation de Méphisto. N'en déplaise à son correspondant Hugo Boxel, Spinoza estime que les spectres ne sont pas nécessaires à la perfection de l'univers. Il juge suspectes les relations les plus fameuses, comme celle du revenant qui, selon Pline le Jeune (Lettres, livre VII, 27), aurait hanté une maison d'Athènes pour exiger une sépulture décente, ou celle des esprits qui, à en croire Suétone (Vie des Césars, livre IV, Caïus Caligula, LIX), se manifestaient dans les jardins de l'empereur défunt. « Je m'étonne [écrit-il], que des hommes intelligents et habiles usent et abusent de leur intelligence pour nous persuader de niaiseries de ce genre ». Quant à l'histoire rapportée par Boxel d'un fantôme qu'on entendrait travailler nuit et jour dans une brasserie, elle ne mérite que le rire (Spinoza, Lettres LI à LVI) ${ }^{1}$. Au sentiment de Lichtenberg, la notion même de spectre n'est guère concevable : «Was ist endlich ein Gespenst? Der Aberglaube antwortet: ein Geschöpf, das um Mitternacht herumkriecht, die Menschen $\mathrm{zu}$ erschrecken; und die Vernunft: ein Ding das mir 100 mal unbegreiflicher ist, als alles unerklärte Rumpeln und Poltern der ganzen Welt» («Etwas über die Poltergeister», Gesammelte Werke hrsg. von W. Grenzmann, BadenBaden, o. J., I, 98). D'ailleurs la plupart des hommes versés dans les sciences de la nature n'attachent aucune valeur aux prétendues preuves de l'existence des spectres : "Allein wo ist der Beweis, daß es Gespenster gibt? Fast alle Männer, deren Werk es ist, sich mit Erforschung natürlicher Dinge abzugeben, leugnen sie oder haben die triftigsten Gründe, die angeblichen Beweise nicht für gültig zu erkennen» (Ibid., 99). Plus 
conciliant, Kant concède que la définition d'un «Geist», être immatériel étendu et pourvu de raison n'enveloppe pas de contradiction, encore qu'elle n'implique pas l'existence. Parlant de l'auteur des Träume eines Geistersehers, c'est-à-dire de, il avoue quelque peu penaud : «er bekennt mit einer gewissen Demüthigung, daß er so treuherzig war, der Wahrheit einiger Erzählungen von der erwähnten Art nachzuspüren. Er fand - wie gemeiniglich, wo man nichts zu suchen hat - er fand nichts» (Werke, Akademie-Textausgabe, II, 318). Plus heureux en un sens, Nicolaï fit savoir au monde savant qu'il avait bien eu des visions, mais que, les tenant pour morbides, il avait su s'en délivrer en se faisant appliquer des sangsues au bon endroit. Nous le retrouvons à la Nuit de Walpurgis :

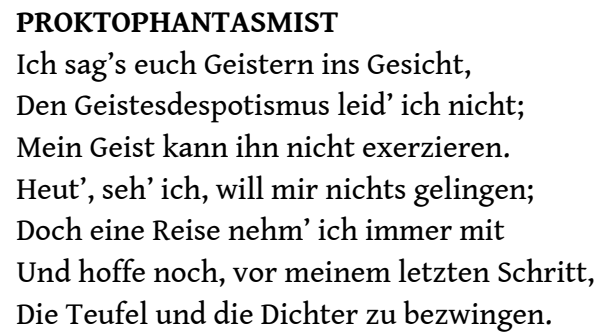

\section{MEPHISTOPHELES}

Er wird sich gleich in eine Pfütze setzen, Das ist die Art, wie er sich soulagiert, Und wenn Blutegel sich an seinem Steiß ergetzen, Ist er von Geistern und von Geist kuriert. Faust I, 4165-4175.

2 Mort ou vif, un philosophe est immuable dans son aspect physique, ses habitudes vestimentaires, son goût des définitions et des spéculations sur les conditions d'existence. Fantôme, il ne croit pas aux fantômes, témoin Saul Ascher, le libraire philosophe dont l'ombre apparut à Heine :

Er sah aus wie sonst, derselbe transzendentalgraue Leibrock, dieselben abstrakten Beine, und dasselbe mathematische Gesicht [...] In seinem gewöhnlichen mundfaulen Dialekte sprach er freundlich: «Fürchten Sie sich nicht, und glauben Sie nicht, daß ich ein Gespenst sei. Es ist Täuschung Ihrer Phantasie, wenn Sie mich als Gespenst zu sehen glauben. Was ist ein Gespenst? Geben Sie mir eine Definition? Deduzieren Sie mir die Bedingungen der Möglichkeit eines Gespenstes? In welchem vernünftigen Zusammenhange stände eine solche Erscheinung mit der Vernunft? Die Vernunft, ich sage die Vernunft -» Und nun schritt das Gespenst zu einer Analyse der Vernunft, zitierte Kants Kritik der reinen Vernunft, 2ter Teil, Ister Abschnitt 2tes Buch, 3tes Hauptstück, die Unterscheidung von Phänomena und Noumena, konstruierte alsdann den problematischen Gespensterglauben, setzte einen Syllogismus auf den andern, und schloß mit dem logischen Beweise: daß es durchaus keine Gespenster gibt.

(Reisebilder. Erster Teil: Die Harzreise,

Sämtliche Schriften II, Darmstadt, 1969, 128)

Or les définitions des fantômes abondent. Celles que les philosophes ont élaborées depuis deux millénaires s'inspirent des traditions populaires autant que des spéculations chaldaïques et néoplatoniciennes. Le dogme chrétien, qui encadre la plupart, n'est pas un obstacle à leur incohérence. Ce point n'a pas échappé à Georg Conrad Horst, qui les expose et les commente dans le premier volume de sa ZauberBibliothek (Mainz, 1821, 236-240). Au sentiment des uns, les fantômes sont les enfants d'un Dieu créateur d'entités réelles, encore que généralement invisibles, supérieures 
aux hommes bien qu'inférieures aux anges. Elles auraient pour mission de rendre des services à l'humanité, de lui inspirer des craintes salutaires et de lui infliger des châtiments mérités. Selon les autres, les fantômes sont des inventions de démons incapables de créer des êtres réels, mais aptes à susciter des apparences trompeuses. Ces fantômes diaboliques s'appliqueraient, par pure malice, à nuire, en les épouvantant, aux hommes et aux bêtes. Une troisième école soutient que les fantômes sont des manifestations tantôt sublimes, tantôt dégradées, de la nature humaine. Les uns voient en eux des bienheureux, ou des âmes du purgatoire, chargés d'apporter quelque message à leurs frères vivants; les autres de simples résidus d'un corps astral, entité formée de matière subtile et vouée comme le corps matériel à la décomposition. Dans cette discipline nourrie de témoignages suspects et étayée d'inférences hasardeuses, que chacun décide selon ses convictions ou ses goûts, à moins qu'il ne préfère suspendre son jugement.

4 Mais sans doute faut-il distinguer, au sein de la philosophie, l'ontologie, qui se prononce sur la nature des êtres, et la phénoménologie, qui s'applique à décrire les apparences. Ayant mis entre parenthèses le problème de l'existence et de la nature des spectres, cette dernière s'efforce d'analyser les impressions de ceux qui se flattent d'avoir l'expérience sensible du spectral ou l'expérience affective du numineux décrites par Lucien Lévy-Bruhl et par Rudolf Otto. Tentons l'entreprise, en nous appuyant sur les deux béquilles que nous proposent l'introspection et la lexicographie. Interrogeons notre conscience en feuilletant, à l'article "Gespenst», le Deutsches Wörterbuch de Grimm.

5 Prenons d'abord le mot «Gespenst» dans son sens le plus populaire, et, si j'ose dire, le plus concret. Voir un fantôme, c'est percevoir une forme, généralement humaine, le plus souvent longue et blanche. Mais cette définition est à la fois trop large et trop étroite. Trop large, parce que je ne qualifierai de fantomatique ni l'image visuelle que j'ai de vous quand vous vous tenez en pleine lumière en face de moi, ni votre reflet que je surprends dans un miroir, ni votre photographie, ni votre image sur un écran de télévision. Trop étroite, parce qu'on parle aussi de fantômes d'animaux, voire d'objets inanimés, et que, si la vue paraît être le sens le plus propre à percevoir les fantômes, ce privilège ne lui est pas réservé. Notre oreille perçoit le son de leurs voix, le bruit des chaînes qu'ils agitent, les notes de leurs mélodies préférées, nos narines reconnaissent leur parfum favori, notre épiderme frissonne au contact de leurs mains glacées. Et, s'il nous plaît de donner le nom de "fantôme " à une image mentale enfantée par la rêverie ou évoquée par la mémoire, nous le prenons dans u $\mathrm{n}$ sens très faible; mais il en va tout autrement lorsque l'image s'impose à nous, avec l'évidence d'une perception sensible, dans notre sommeil. Il arrive même qu'un être invisible se manifeste par ce "sentiment de présence » que William James a décrit dans ses Variétés de l'expérience religieuse (the Varieties of Religious Experience, Lecture III ; «The Reality of the Unseen»).

6 Autre sujet de perplexité : ne faut-il pas distinguer le fantôme tout court du fantôme de quelqu'un ? Le premier seul jouirait à coup sûr d'une existence autonome. S'il existe des fantômes des vivants ou des morts, où réside leur moi ? Est-ce dans leur corps de chair pour les premiers, dans l'au-delà pour les autres ? N'est-ce pas plutôt dans le local où se manifeste leur présence fantomale?

7 Autre problème : faut-il appliquer au fantôme, en lui donnant son sens le plus fort, la formule de Berkeley : esse est percipi, être, c'est être perçu ? La tradition s'y refuse : nés de nos craintes ou de nos désirs, les fantômes mourraient avec eux. De plus, le fantôme 
diffère du personnage que nous voyons évoluer sur un écran de télévision en ceci que, non content d'être vu, il nous voit aussi. Il nous observe par ses yeux, nous parle par sa voix, attire notre attention par ses gestes, manifeste ses intentions par son comportement. Etre, pour un fantôme, c'est percevoir autant qu'être perçu : esse est percipi et percipere. Mais certains vont plus loin: être autonome, le fantôme n'a pas besoin de notre perception pour exister. Établi dans un lieu hanté, il ne se manifeste que pour effrayer les visiteurs importuns et jouir seul de sa demeure. Ne faut-il pas en fin de compte reprendre la formule complète de Berkeley: être, c'est être perçu ou percevoir : esse est percipi aut percipere ? Décide qui pourra.

8 Le «Gespenst» ainsi décrit est celui des «Volkssagen» du folklore et des «Gespenstergeschichten» de la littérature. C'est la «schattenhafte Erscheinung» de l'âme errante d'un défunt («von einer abgeschiedenen Seele»), d'un démon, d'un farfadet ou d'un lutin («Irrwisch», «Kobold»), c'est un esprit qui hante un lieu («umgehender Geist») ou qui y mène grand tapage («Poltergeist»), voire une image onirique («Traumbild») qui, loin d'être un fantasme du dormeur, manifeste une présence étrangère.

Or, réel ou imaginaire, fantôme véritable ou simple épouvantail («Schreckbild»), vision atmosphérique inquiétante («unheimliche Lufterscheinung») ou image trompeuse flottant devant les yeux «phantastisches Bild vor den Augen»), voire animal ou plante à l'aspect étrange ou inquiétant («als Name von Tieren und Pflanzen wegen ihres seltsamen Aussehens oder unheimlichen Auftretens»), le «Gespenst» est avant tout une vision qui sème l'effroi («eine Schrecken und Grauen erregende Erscheinung»). Un trait de caractère se détache de l'objet qui était supposé le susciter et s'applique aux choses les plus diverses.

Chose effrayante encore que faite d'ombre et non de chair, réalité permanente dans son être encore que fugace dans ses manifestations, le «Gespenst» cesse d'être prestigieux quand on le tient pour illusoire. Le terme s'applique aux expériences les plus banales et les plus familières, les plus innocentes aussi, parce que, incapables de séduire la croyance, elles ne sont même pas en mesure d'égarer les sens. Un fantôme, c'est par exemple une image virtuelle ou une image consécutive. Goethe prend «Gespenst» dans le sens de «Spectrum, Scheinbild». Il forge le terme d'«Augengespenst», dit-il, «weil man dasjenige was erscheint ohne Körperlichkeit $\mathrm{zu}$ haben, dem gewöhnlichen Sprachgebrauche nach ein Gespenst nennt». Toutefois, la pensée glisse aisément de l'impression inconsistante et fugitive qui se dissipe sitôt née à la tromperie diabolique en passant par l'illusionnisme et la magie. Le «Gespenst» est pure apparence, ombre, image trompeuse («bloßer Schein», «Schatten», «Scheinbild»), ennemi du vrai, du réel, de l'authentique («das Wahre, Wirkliche, Echte»). C'est l'illusion menteuse («Blendwerk», «Täuschung», «Trug») produite par un prestidigitateur («Gaukler»), un magicien («Zauberer»), voire «ein böser Geist». Ouvrage du démon qui induit en tentation, le «Gespenst» est «Verlockung, Verführung, Versuchung». Selon Luther, le Séducteur fait briller devant les yeux de ses dupes des trésors illusoires: «Im Bergwercke vexiret und betreuget der Teufel die Leute, macht jhnen ein Gespenste und Geplerr für den Augen, daß sie nicht anders wehnen, als sehen sie ein großen Hauffen ertztes und gediegen Silber, da es doch nichts ist». En somme, qu'il signifie une réalité ou une illusion, le mot «Gespenst» est presque toujours pris en mauvaise part. Ce n'est qu'exceptionnellement qu'il désigne une inspiration («Eingebung») de l’Esprit saint. 
11 Les doubles des vivants qui s'évadent de leur prison charnelle s'apparentent aux fantômes des morts. Les uns et les autres sont aussi mystérieux qu'insaisissables. Le même médium qui extériorise sa sensibilité prête aussi une apparence évanescente aux âmes des défunts. Or, parmi ces dernières, celles des élus et celles des damnés se rapprochent, qui des anges bienheureux, qui des démons de l'enfer, purs esprits frères par leurs origines, encore qu'ennemis irréconciliables par le choix de leur conduite. L'occultiste Carl du Prel est bien conscient de ces affinités : Un même corps astral se manifeste de manière ou spontanée ou provoquée, ou dans la vie ou dans la mort. Il importe peu d'ailleurs que le sujet prenne l'initiative de sa manifestation, ou qu'elle lui soit imposée :

Die Trennbarkeit des Astralleibes vom Körper ist denkbar:

1. Im Leben.

a) Als unwillkürliche Trennung: Doppelganger.

b) Als willk ürliche Trennung: Majavi-Rupa.

c) Als Trennung durch fremden Willenszwang: Citation.

2. Im Sterben.

3. Nach dem Tode.

a) Als willkürliche Darstellung der Astralleiber: Gespenster.

b) Als veranlaßte Darstellung: Materialisation; Nekromantie. (Die monistische Seelenlehre, Leipzig, 1888, 170).

Peu importe aussi que le double apparaisse aux yeux du sujet ou à ceux d'autrui :

a) daß der Mensch seinen eigenen Doppelgänger sieht,

$ß)$ daß der Doppelgänger in der Entfernung von anderen gesehen wird. (Ibid., 171).

Dans ses Mystères d'Égypte, Jamblique mettait en garde les adeptes de la théurgie contre des méprises fâcheuses : les êtres qu'ils évoquent ne déclinent pas toujours leur identité véritable: il n'est pas rare que des entités d'ordre inférieur se fassent passer, par vantardise, pour des esprits supérieurs. Et l'avertissement de Paracelse aux nécromanciens de son temps vaut pour les spirites du nôtre : pensant s'entretenir avec un mort, ils conversent en fait avec un diable qui a pris possession de son corps astral.

15 Ces remarques incitent à prendre le mot « spectre » dans son sens le plus large. Quant aux philosophes, ils s'évertuent depuis plusieurs millénaires à se définir eux-mêmes. Parmi la gamme des définitions qu'ils ont proposées, je retiendrai celle-ci: le philosophe, c'est l'homme qui dédaigne les agréments de la fiction pour s'adonner à la recherche de la vérité, c'est le "théoricien », «der theoretische Mensch» de Spranger. Ce choix m'autorise à prendre en considération, avec les opinions de philosophes au sens strict du terme comme Schopenhauer, celles de médecins comme Paracelse ou d'occultistes comme du Prel.

\section{II}

La croyance au corps astral est des plus anciennes ${ }^{2}$. Une longue tradition, folklorique, religieuse et philosophique veut que l'homme ne soit pas double, mais triple. Pendant que le corps du défunt se décompose dans la tombe et que son âme prend son essor vers le ciel, son fantôme continue à hanter la terre. Et cet être est dangereux : «die Toten töten», dira Freud. Deux sentiments se partagent l'âme de ceux qui participent aux 
veillées funèbres : ils pleurent le départ d'un parent ou d'un ami, ils craignent de voir le cadavre s'animer. Or, ce qu'ils redoutent, ce n'est pas le retour de l'âme du défunt, c'est un spectre, un «mort vivant». Généralement invisible, le corps astral se manifeste quelquefois, c'est le double, dont l'apparition passe pour un présage funeste.

Cependant, chez Platon et surtout chez les néo-platoniciens, cette troisième entité n'est pas redoutable. Elle est le vêtement intérieur de l'âme caché sous ce vêtement externe qu'est le corps charnel. Elle est le véhicule que l'âme emprunte pour descendre sur la terre, remonter au ciel ou communiquer avec d'autres âmes.

Une tradition judéo-chrétienne veut que Dieu seul soit purement immatériel. Les anges seraient dotés d'un corps subtil, qui leur permet de se montrer parmi les hommes, d'accepter les aliments que leur offrent leurs hôtes, et d'exciter la convoitise des Sodomites. Et l'on s'expliquerait mal que les damnés puissent endurer des tourments physiques s'ils n'étaient dotés d'un corps.

Ce dernier est qualifié d'astral, parce qu'il serait composé, comme les corps célestes, d'une matière plus fine («feinstofflich») que celle qui compose les corps terrestres, d'une «quinta essentia», plus subtile que les quatre autres : la terre, l'eau, l'air et le feu (voir P. Moraux, «Quinta essentia» in Realencyclopädie de Pauly, Wissowa...).

Cependant la pensée chrétienne tendait à refuser l'existence à cette entité intermédiaire, et professer un dualisme qui revêtira sa forme la plus nette et la plus brutale chez Descartes : L'homme est formé par l'union d'une âme, pensée pure, et d'un corps, étendue matérielle. Or ces deux substances sont si étrangères l'une à l'autre qu'on est obligé, pour expliquer leur union et leur interaction, de forger des hypothèses aussi bizarres que hasardeuses. La réaction ne se fait pas attendre: étrangère à l'étendue, l'âme cartésienne n'est nulle part. C'est pourquoi Glanvill et son ami Henry More voient en Descartes «le prince des nullibistes ${ }^{3}$ " - À leur sens, tout esprit est étendu et localisé dans l'espace. Quant à Leibniz, que l'on considère à tort comme cartésien, il croit «avec la plupart des anciens que tous les génies, toutes les âmes, toutes les substances simples et creées sont tousjours joints à un corps, et qu'il n'y a jamais des âmes entièrement séparées " (Nouveaux essais..., Préface/Die philosophischen Schriften hrsg. von Gerhardt, V, 51). S'il soutient, dans sa Monadologie, qu'il n'existe ni métempsycose ni migration des âmes, «il n'y a pas non plus des Âmes tout à fait séparées, ni des Génies sans corps. Dieu seul en est détaché entièrement » (\$ 72).

De nos jours, occultistes et spirites croient volontiers à l'existence du corps astral de leurs parents et de leurs amis, mais bien éloignés d'en craindre les manifestations, ils les désirent et les provoquent. À leur sentiment, les êtres qui manifestent leur présence par des «messages » et des "matérialisations » ne sont pas des spectres menaçants, mais des âmes bienveillantes.

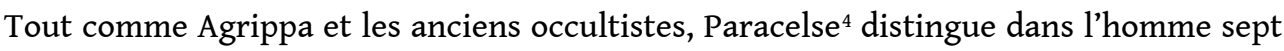
parties fondamentales :

23 1) Le corps élémentaire visible dont les os, la chair et le sang sont formés d'éléments solides, fluides et gazeux ; 
2) L'âme végétative («anima vegetativa», «spiritus vitae»), fluide ou force vitale qui anime le corps matériel ;

3) L'homme sidéral, corps astral ou evestrum, composé des essences subtiles et des effluves provenant des astres de notre système solaire ;

4) L'esprit animal, ou âme astrale, dont le rôle est d'animer le corps astral, comme l'âme végétative fait le corps élémentaire ;

5) L'âme raisonnable, dotée d'entendement ;

6) L'âme spirituelle, siège de la conscience morale et des aspirations les plus hautes. Cette dernière est à son tour en relation étroite avec l'âme raisonnable ;

7) L'homme de l'olympi novi, indépendant de l'homme terrestre, dont il contrôle les fonctions.

Ce schéma est assez simple : l'homme est composé de trois paires d'éléments, assurant respectivement sa vie physique, sa vie psychique, et sa vie spirituelle. Le premier élément de chaque paire est soumis au second. Le septième élément contrôle l'ensemble ${ }^{5}$.

31 Alors que les auteurs s'accordent assez bien sur la nature des choses, ils divergent souvent dans l'emploi du vocabulaire. Tel auteur fait de l'âme («Seele») une entité supérieure à l'esprit («Geist»), tel autre fait le choix inverse. D'autre part, chez Paracelse lui-même, « corps astral » est un concept assez élastique. Au corps astral du minéral, "stannar» ou "truphat", véhicule d'une force aveugle, s'ajoutent successivement, chez les animaux, une entité appelée «leffas», qui gouverne la vie végétative, puis une autre nommée «evestrum», organe de la vie sensorielle et mentale (voir Martin Ruland, Lexicon Alchemiae, Francfort, 1612).

En maints endroits, Paracelse insiste sur la distinction du corps matériel issu d'Adam, partant du limon, et du corps astral, seul promis à la résurrection. Différent en cela du premier, le second échappe aux servitudes imposées aux réalités physiques :

Das Fleisch muß so verstanden werden, daß sein zweierlei ist, das Fleisch aus Adam und das, so nit aus Adam ist. Das Fleisch aus Adam ist ein grob Fleisch, denn es ist irdisch, und ist sonst nichts als allein ein Fleisch, das zu binden und zu fassen ist wie ein Holzoder Stein. Das ander Fleisch, das nit aus Adam ist, das ist ein subtil Fleisch, und ist nit zu binden noch zu fassen, denn es ist nit aus der Erde gemacht. Nun ist das Fleisch aus Adam der Mensch aus Adam, der ist grob wie die Erde (die selbige ist kompakt) so daß der Mensch nit durch eine Mauer noch durch eine Wand kann, er muß sich ein Loch machen, durch das er schlieft, denn ihm weicht nichts. Aber das Fleisch, das nit aus Adam ist, dem weicht das Gemäuer; das ist: die selbigen Fleische bedürfen keiner Tür, keines Lochs, sondern gehen durch ganze Mauern und Wände und zerbrechen nichts.

(Liber de nymphis, Caput secundum / Peuckert III, 467)

Normalement invisible, le corps astral apparaît parfois après la mort :

Spectrum. Das sind nun die Gesichte, die die astralischen Körper von dem Menschen, der gestorben ist, geben. Denn ein Mensch hat zween Körper, einen von den Elementen, den andern vom Firmament. Der von den Elementen stirbt und fault in der Erden, das ist der, der da vergraben wird; der andere schwebt in den Lüften und auf Erden usw.; wo nun solche corpora aetherea von einem gestorbenen Menschen gesehen werden, das ist ein spectrum.

(Herbarius, «Von den Korallen» / Peuckert I, 281)

Paracelse développe avec insistance la thèse suivante : le corps astral n'est pas, comme beaucoup l'imaginent, quelque âme humaine errante ou quelque esprit autonome. 
Cette entité, qui n'occupe qu'un degré inférieur dans l'échelle des parties qui constituent l'être humain, n'est qu'un corps - et après la mort, qu'un cadavre - subtil. $\mathrm{Au}$ reste les deux corps connaissent des destins semblables; la vie les unit, puis la mort les sépare et les voue l'un et l'autre à la destruction. Chacun retourne aux éléments dont il a été formé : éléments matériels pour l'un, effluves astraux - «Gestirn» - pour l'autre, et chacun se décompose dans son milieu naturel, qui dans la terre, qui dans l'atmosphère :

So verzehrt sich der elementierte Leib in den Elementen, [...] aber von dem siderischen Leibe wisset seine Fäulung so: er ist vom Gestirn und nicht von den Elementen, drum hat er seine Verzehrung nicht in den Elementen, sondern außerhalb der Elemente, das ist: unter dem Gestirn, und muß gleichwohl mit der Zeit verzehrt werden wie der elementierte Leib, von dem, in das er vergraben wird, das ist: vom Gestirn, wie der elementierte Leib von den Elementen. Nun folgt darauf, daß der siderische Leib bei dem Körper bleibt, so lange, bis er auch von dem Gestirn verzehrt wird. Das ist: wie sie im Leben mit einander vermählt gewesen waren, so werden sie durch den Tod geschieden, ein jeglicher in sein Grab der Verzehrung. Sie bleiben jedoch beieinander, der eine in den Elementen, der andere außerhalb der Elementen in der Luft, und in der Luft ist sein Gewaltiger, das ist: in der Luft verzehrt ihn das Gestirn. So verzehrt die Erde den elementierten Leib und das sidus den siderischen, und so nehmen beide Leibe ihre Consumation. Nun bedarf der elementierte Leib einer Zeit, bis er verfault, einer mehr denn der andere. So hat auch der siderische Leib eine solche Zeit. Und wie es sich genügend beweist daran, wie die Leibe in den Elementen verzehrt werden, so muß auch der siderische Leib eine Zeit haben, bis er auch verzehrt werde. Der elementierte Leib ist greiflich, der siderische Leib aber ist nicht greiflich, sondern wie ein Geist. Also wird der elementierte Leib greiflich gesehen, der siderische ungreiflich.

(Philosophia sagax, 1. Buch, 6. Kap.,

Probatio in scientiam nigromanticam / Peuckert III, 162-163).

Si étrange et fantastique qu'elle paraisse à première vue, cette conception est cohérente et, tout compte fait, rationaliste, ennemie des croyances superstitieuses. Loin d'être un esprit qui hante les ténèbres, le corps astral d'un mort n'est qu'un cadavre subtil, aussi vide d'intentions et de connaissances qu'un cadavre matériel.

C'est pourquoi si je dis :

... ich hab dessen Geist gesehen! Ich hab den gehen gesehen, so es doch nur der siderische Leib ist, der so sein Vergräbnis und seine Verzehrung hat. Und es ist übel geredet, daß man sagt und glaubt, es sei der selbige Mensch, als wäre er gar und endlich gar vollkommen da, so es doch keines ist, auch keine Seel, auch der selbige Mensch nit, sondern allein ein siderischer Geist. Gleicherweis als wenn der elementierte Leib nicht vergraben wäre, so könnte er gesehen werden, jedoch so ist es der selbige Mensch nit, aber wohl ein Stück von ihm, ein Teil von ihm, das da ohne Leben ist, tot und im Grab. So wird der siderische Leib gesehen, denn er kann nicht vergraben werden, denn er ist nicht greiflich, sondern ein Geist, wie ein Bild im Spiegel. Nun ist zwar der siderische Leib auch tot...

(Ibid., 163-164)

Partant de ces prémisses, Paracelse réfute trois thèses, à savoir celles des «Exorcisten», des «Conjuristen» et des «Totenbüchler». Prenant le corps astral pour un esprit, les premiers se flattent de s'entretenir avec lui et de l'obliger à répondre à leurs questions. Or, ce n'est pas à un être vivant qu'ils s'adressent, mais à une chose morte, incapable d'entendre et de parler. Les seconds, qui se targuent de conjurer le corps astral et de le plier à leurs désirs, ignorent que l'homme n'a pas ce pouvoir. Et ces êtres frivoles font le jeu du diable qui, capable de posséder un vivant, est à plus forte raison en mesure de s'introduire dans une chose morte qui n'est pas en état de lui résister. Ce n'est donc pas 
à un être humain qu'ils ont affaire, mais à un démon. Les troisièmes voient dans les « esprits » des âmes du purgatoire ou de l'enfer tenues d'expier leurs fautes jusqu'à la fin des temps, et qui supplient les vivants d'intercéder pour elles afin qu'elles puissent gagner le ciel. Ils ignorent que les âmes des défunts ne résident ni dans la terre ni dans l'atmosphère, mais dans un lieu inaccessible, qu'elles ne quitteront qu'au jour du jugement dernier, et qu'il est aussi vain de prier pour un corps astral que pour un cadavre (Ibid., / Peuckert III, 165-166).

Comme on voit, Paracelse, à l'instar de ses contemporains, croît à l'existence et à l'activité des diables que d'aucuns prennent pour les « esprits » des défunts. Or, sachez que, quand ces derniers semblent mener grand tapage en poussant des cris de guerre, ce sont des diables qui se réjouissent à la pensée qu'ils ont gagné la partie, que le sang va couler en abondance, en sorte qu'il est temps que chacun s'amende et fasse pénitence :

... wo solche Rumpelgeister wie mit Kriegsgeschrei gehen, da ist ein groll Blutvergiellen vorhanden, dess' freuen sich die Teufel, lachen und spielen es zuvor, eine Zeit Jang, als sprachen sie: wir haben da ein gewill Spiel. Sehe ein jeder, wer da praefiguriert wird, auf sich selbst, daß er sich bessere und leide Bulle.

(Liber de animabus hominum post mortem apparentibus,

V / Peuckert IV, 381).

Ainsi, ces diables étourdis donnent des armes à leurs adversaires. Mais chacun sait que les démons occupent une place importante dans les exposés populaires d'apologétique et de morale. Et les apparitions des morts sont, elles aussi, lourdes de signification. L'attitude du mort laisse entrevoir le destin promis au vivant. Est-elle amicale, vous compterez parmi les élus. Est-elle effrayante ou hostile, elle vous incite à mettre à profit votre vie terrestre pour accorder vos pardons et payer vos dettes. Puisse leur aspect terrifiant vous mettre en garde contre le sort qui pourrait vous être réservé dans l'au-delà :

Wenn ein Toter gesehen wird, so denke daran, daß er ein praesagium ist. Und das so : steht er da freundlich, wie ein Heiliger, so ist das soviel, als spräche er: ihr seid der Seligen. Steht er aber anderwegs da, so ist soviel, daß er noch nit abgerechnet und bezahlt hat; er muß auf die Zeit der Verzeihung und Vergebung oder Bezahlung seiner Kunden warten. Wenn er drum so steht, so ist es ein Anzeichen, daß wir es uns ein Exempel sein lassen, je einer dem andern zu vergeben, und die harte Erscheinung, die so erschrecklich ist, ernsthaft betrachten.

(Ibid., / Peuckert IV, 379)

40 Je hasarderais cette interprétation: illusoires dans leur réalité, les manifestations d'outre-tombe ne laissent pas, tout comme les fables édifiantes et les paraboles, d'avoir une portée religieuse ou morale, d'autant plus que, frappant fortement les sens et l'imagination, elles n'en sont que plus efficaces. Un psychologue moderne pourrait considérer ces visions comme des projections de la conscience morale dans un uni vers fantastique.

41 S'il faut en croire des auteurs graves, les premiers incubes sont apparus avant le Déluge. D'après la Genèse (VI, 4), « en ces jours-là il y avait des géants sur la terre et même après cela : quand les fils d'Elohim venaient vers les filles des hommes et qu'elles enfantaient d'eux, c'étaient les héros qui furent jadis des hommes de renom » (trad. 
E. Dhorme). Que faut-il entendre par là ? Saint Augustin estime que les filles de Caïn ont séduit les fils de Seth (Cité de Dieu, livre XV, chap. 8). Mais d'autres commentateurs pensent qu'il faut prendre le texte sacré dans son sens littéral: des anges dépravés auraient fait aux filles des hommes des enfants vigoureux, des géants. Or, il est clair que ces esprits paillards ne pouvaient être que des diables. Saint Thomas aborde très brièvement la question dans sa Somme théologique (Ia 51, 3, § 6). À son sentiment, les diables sont incapables de produire de la semence humaine, en sorte que l'enfant issu du commerce d'un diable et d'une femme aurait pour père, non pas le démon, mais l'homme dont il aurait capté le sperme. Le problème n'était pas tranché à la fin du XVI ${ }^{\mathrm{e}}$ siècle, quand le grand démonologue Bodin résume les débats en ces termes :

... les Docteurs ne s'accordent pas en cecy : entre lesquels les vns tiennent que les Demons Hyphialtes, ou Succubes, reçoiuent la semence des hommes, \& s'en seruent enuers les femmes en Demons Ephialtes, ou Incubes, comme dict Thomas d'Aquin, chose qui semble incroyable: mais quoy qu'il en soit, Sprenger escrit que les Allemans qui ont plus d'experience des Sorciers, pour y en auoir eu de toute ancienneté, \& en plus grand nombre qu'és autres pays, tiennent que de telle copulation il en vient quelquesfois des enfans, qu'ils appellent VVechselkind, ou enfans changez, qui sont beaucoup plus pesans que les autres, \& sont tousiours maigres : \& tariraient trois nourrices sans engresser. Les autres sont diables en guise d'enfans, qui ont copulation auec les nourrices Sorcieres, \& souuent on ne sçait qu'ils deuiennent. Mais quant à telle copulation auec les Dernons sainct Hierosme, sainct Augustin, sainct Chrysostome, \& Gregoire Nazienzene, soustiennent contre Lactance, \& Joseph, qu'il n'en prouient rien, \& s'il en vient quelque chose, ce serait plustost vn Diable incharné qn'vn homme.

De la demonomanie des Sorciers, Paris, 1587,

livre second, chap. VII, fol. $118 \mathrm{r}^{\circ}$ et $\mathrm{v}^{\circ}$.

Cependant la question avait été traitée avec toute l'ampleur désirable dès 1487, par Jakob Sprenger et Heinrich Institoris dans leur fameux Malleus Maleficarum ou Hexenhammer. Il n'est pas douteux, d'après eux, que le diable s'accouple souvent avec ses servantes dans les champs et dans les bois, comme chacun peut juger d'après le comportement des dames, le corps de leur amant étant généralement invisible ${ }^{6}$. Sur le fond, ils s'accordent avec saint Thomas. Et, à les en croire, ces diables fornicateurs auraient inventé, avec l'eugénique, la fécondation artificielle. Dédaignant le sperme répandu à la faveur des pollutions nocturnes, semence affaiblie que l'organisme rejette pendant le sommeil, ils captent celui qui est émis au cours du commerce charnel. Il semble qu'un diable succube confie à un collègue incube le soin de porter à destination la semence recueillie. Comme les démons sont très versés dans la connaissance des êtres vivants et des astres, ils confient à la meilleure des destinataires la semence du meilleur des donneurs au meilleur moment possible. Il n'est donc pas étonnant que ce commerce diabolique produise des rejetons vigoureux, ces géants dont parle la Genèse. On va voir que Paracelse professe une théorie fort différente, et selon laquelle les fruits de l'incubat s'apparentent aux Wechselkinder maigrichons et stupides du folklore plus qu'aux géants vigoureux de la Genèse (Malleus maleficarum, II, Q.I. Cap. IV).

Il la développe au troisième livre de son ouvrage De cousis morborum invisibilium consacré à l'étude de la faculté qu'il appelle en allemand «Imagination », et qui diffère fort d'une innocente "Phantasie ». Il se flatte, dans le prologue, d'être le premier à en traiter selon les principes de la philosophie : «Obwohl bisher keiner aus dem Grunde der Philosophie diese Dinge angerührt hat, so werde ich mich nicht schamen, hierin, [...] der erste zu sein» (Peuckert II, 220). Il se fait fort de rendre compte, grâce aux lumières de l'intelligence et aux données de l'observation, au mépris de toute tradition, 
superstition, «Zauberei, Gespensterei und Geisterei», de phénomènes réputés prodigieux.

L'imagination est en effet capable de gouverner le corps astral, partant de modeler le corps élémentaire. Il en donne des exemples multiples, dont les plus frappants sont relatifs à la grossesse. Une femme pose sur son genou un limaçon qui il frappé sa vue ; aussitôt l'image de la bête se dessine sur le genou de l'enfant qu'elle porte. Il est clair que le corps physique de la mère vient d'obéir à une injonction de son imagination, tout comme fait un paysagiste quand il exécute l'ordre de peindre un limaçon sur une branche de son tableau (224). C'est pure superstition de croire que le diable dessine et colore les taches de naissance.

Or c'est l'imagination des luxurieux qui est à l'origine de la croyance aux succubes et aux incubes; c'est elle qui produit le sperme qu'on prétend véhiculer par le diable. Au reste, ce n'est pas un sperme naturel, mais, selon une expression que Paracelse emprunte à la Bible de Luther (Mtt., V, 13 ; Mc., IX, 50 ; Le., XIV, 34), un sel affadi, «ein dummes Salz» :

Es ist auch wahr, daß die Imagination ein Ursprung der succubi und incubi ist, dergestalt daß die Imagination die Dinge gebiert und alsdann vertragen wird. Um dieses zu verstehen, muß zu allererst das sperma incubi und succubi verstanden werden, von wannen dieses sperma komme und geboren werde. Es kommt aus der starken Imagination aller derer, die in Sinnen und Gedanken buhlen. Alldieweil es nun aus der Imagination kommt, so ist es kein rechtes natürliches sperma, sondern wie ein dummes Salz. (234)

En prenant «ein dummes Ding für ein vollkommenes», Thomas d'Aquin est dans l'erreur (235). En fait, ce faux sperme ne provient ni des pollutions involontaires, ni des relations conjugales, mais de l'imagination déréglée de ceux qui s'adonnent à des pratiques contre nature :

Nun aber ist dieses sperma, das aus der Imagination kommt, in amore hereos ${ }^{7}$ geboren. Was ist aber diese selbige Liebe? Nichts anderes als allein die, die da im Sinnen sich selbst eine Frau phantasiert, und so diese selbige Buhlschaf $t$ auf ein Ende führt. Dazu entspringt dann das Ausgehen eines dummen tauben spermatis, das da nicht voll kommen ist Kinder zu gebären; das selbige sperma ist aber das, das hier incuburn und succubum gebiert. (235)

Et comme ce n'est pas la nature, mais l'imagination qui gouverne ce sperme, il est la cause principale de la stérilité, et l'origine des faux germes. Il ne semble pas d'ailleurs qu'il soit de nature essentiellement masculine, car c'est lui qui provoque ces grossesses nerveuses qu'on rencontre même chez des femmes qui ne sont plus en état de procréer.

Mais il y a pire : recueilli par les « esprits de la nuit » et incubé par des bêtes impures, le «dummes sperma» est capable de produire des monstres:

Damit ich aber weiter von der selbigen Gebärung incubi und succubi rede, so wisset, daß das selbige sperma durch die Geister, die zur Nacht wandeln, vertragen wird. Die tragen es an Enden und an Örter, da es ausgebrütet werden kann, zum Beispiel unter die Würmer, Krüten und dergleichen unreine Tiere. Denn da geschieht von den Nachtgeistern an solchen Tieren, auch an Hexen, mit diesem sperma ein actus, daraus dann viel seltsamer monstra, deren eine Zahl ist, die aber doch im Angesicht unserer Augen schrecklich sind, geboren werden. $(235)^{8}$

Or ces Nachtgeister ne ressemblent pas plus aux diables avisés des théologiens que les produits de leurs «actes» ne s'apparentent aux géants de la Genèse ou du Malleus maleficarum. Paracelse ne leur concède qu'une existence amoindrie, proche de celle des êtres engendrés par l'imagination : 
Phantasma. Das sind Nachtgeister, die natürlicher Art sind, und die etwas menschlichen Verstandes haben und den Menschen suchen, der ihnen darin verfügbar ist. Deren sind viel, gute und böse, aber nit sichtbar, und sind bei den Menschen wie Hunde, die den Menschen auch lieben oder um ihn sind [...] Und sind gleich wie Incubus und Succubus, Gestalten, die aus dem Phantasieren entstehen. Herbarius, Von den Korallen / Peuckert 1, 280-281.

III Certains l'accusent d'être secrètement athée : il se serait rallié au théisme parce qu'il aurait redouté le pouvoir politique, ou qu'il se serait bercé d'illusions consolatrices. Une page brillante de Heine nous montre le philosophe placide de Känigsberg prenant le ciel d'assaut, passant ses habitants au fil de l'épée, sans épargner le maitre des lieux : «der Oberherr der Welt schwimmt unbewiesen in seinem Blute, es gibt jetzt keine Allbarmherzigkeit mehr, keine Vatergüte, keine jenseitige Belohnung für diesseitige Enthaltsamkeit...» (Zur Geschichte der Religion und Philosophie in Deutschland, drittes Buch, op. cit., Bd III, 604). Alors, se saisissant de la Raison pratique comme d'une baguette magique, il aurait ressuscité le cadavre du théisme tué par la Raison pure. En fait Kant était plutôt fidéiste : que nous ne puissions démontrer l'existence de Dieu ne doit en rien nous détourner de croire et d'espérer en Lui. Parmi nos contemporains, une disciple de Carl Gustav Jung, Aniela Jaffé rapproche la pensée de Kant de celle de l'inventeur des archétypes (Geistererscheinungen und Vorzeichen, Zürich und Stuttgart, Rascher, 1958, 210-213). L'occultiste Carl du Prel, homme de vaste culture, soutient que Kant n'aurait pas manqué de se rallier à ses vues s'il avait eu connaissance du somnambulisme et du spiritisme («Kants mystische Weltanschauung», in Carl du Prels Ausgewdhlte Schriften, L Bd, Leipzig, E. Günther, 1900). Il est indéniable que Kant croyait à la survie et à la communication des âmes dans l'au-delà comme tous les philosophes chrétiens ou platoniciens. Mais, s'il fallait qualifier de «mystiques » tous ces penseurs, le terme perdrait toute précision.

51 Le passage favori des adeptes de l'interprétation mystique de Kant figure dans un ouvrage posthume publié à Erfurt en 1821 à partir de cahiers de cours par Karl Heinrich Ludwig Pötzl : Vorlesungen über die Metaphysik. Il tend à démontrer que l'opposition kantienne du monde sensible et d'un monde intelligible correspond à celle que Swedenborg établit entre l'en-deçà et l'audelà, en sorte que, en dépit des apparences, les deux auteurs sont d'accord sur l'essentiel. Le voici :

Der Gedanke des Swedenborg ist hierin sehr erhaben. Er sagt: die Geisterwelt macht ein besonderes reales Universum aus; dieses ist der «mundus intelligibilis», der von diesem «mundo sensibili» muß unterschieden werden. Er sagt: Alle geistige Naturen stehen mit einander in Verbindung; nur die Gemeinschaft und Verbindung der Geister ist nicht an die Bedingung der Körper gebunden; da wird nicht ein Geist den andern weit oder nahe seyn, sondern es ist eine geistige Verbindung. Geistersehers erläutert durch Träume der Metaphysik, essai que Kant rédigea en 1765, qu'il publia l'année suivante, et que les esprits superficiels tiennent pour un simple pamphlet.

Ces derniers ont des excuses. Dans un texte qu'il écrit de sa main, qu'il fait publier puis rééditer, et qu'il n'a jamais renié que je sache, Kant adresse à «Herr Schwedenberg» les 
compliments suivants : ce «Schwärmer», qui se flatte d'être le prince des vision naires («Erzgeisterseher unter den Geistersehern») est aussi le prince des rêveurs extravagants («Erzphantast unter den Phantasten») (II, 354). Il a publié, sous le titre d'Arcana caeleslia, «acht Quartbände voll Unsinn [...] Der Stil des Verfassers ist platt. Seine Erzählungen und ihre Zusammenordnung scheinen in der That aus fanatischem Anschauen entsprungen zu sein...» (II, 360). Kant se déclare «müde die wilden Hirngespinste des ärgsten Schwärmers unter allen zu copiren» (II, 366). Au reste, un scrupule le retient d'en dire davantage. De même que les faux germes (Miflgeburten) exhibés par les collectionneurs de curiosités naturelles ont le plus fâcheux effet sur les femmes enceintes, des citations de Swedenborg pour raient inciter certaines cervelles à concevoir des avortons ou des monstres (Mondkälber) (II, 366). Que celui qui désire se renseigner et qui a sept livres sterling à gaspiller se procure le texte original !

Chacune des opinions recèle une parcelle de vérité : Kant a bien brocardé Swedenborg, mais l'essai de 1766 est un ouvrage sérieux où Kant esquisse les thèmes qu'il développera dans ses grands livres. D'autre part, très semblables dans leur formulation, certains passages de Swedenborg et de Kant revêtent des significations différentes. Il en est de Swedenborg comme de l'Ecriture sainte : ridicule quand on la prend à la lettre, telle page est acceptable, sinon sublime, quand on la prend au sens figuré.

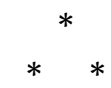

55 Bien avant 1765 , le philosophe s'était intéressé aux prodiges attribués au visionnaire. En témoigne la longue lettre qu'il adresse, le 10 août 1758, à Charlotte von Knobloch. L'un de ses amis, qu'il avait prié de se mettre en relation avec Swedenborg, s'était d'abord montré circonspect. Il changea d'avis quand Swedenborg le reçut dans sa maison; il avait affaire à un homme raisonnable, encore que persuadé que Dieu lui avait accordé le don de communiquer à loisir avec les âmes des défunts. Plus tard, Kant fit tenir à Swedenborg une lettre à laquelle ce dernier promit une réponse qui tarda à venir. Sollicité à nouveau, le visionnaire informa son correspondant qu'on trouverait tous les éclaircissements souhaitables dans un ouvrage qui paraitrait incessamment.

Kant expose à sa correspondante trois anecdotes dignes d'intérêt. Selon la première, Swedenborg aurait fait part à la reine de Suède stupéfaite, de précisions qu'elle était seule à connaître, mais qu'il tenait de ses correspondants de l'au-delà. La chose était attestée par les personnages les plus graves et les plus importants. Selon la seconde, cédant à la demande d'une veuve, $\mathrm{M}^{\mathrm{me}}$ Harteville - que Kant appellera plus tard Marteville -, Swedenborg se serait entretenu avec le défunt mari, qui lui aurait révélé la cachette où il avait rangé un document important. Selon la troisième, Swedenborg, alors à Güteborg, avait eu révélation qu'un incendie venait d'éclater à Stockholm et qu'il menaçait sa maison, puis que le sinistre avait été maîtrisé. Or il s'avéra que ces propos, qu'il avait tenus devant plusieurs témoins au moment précis des faits, étaient parfaitement exacts.

57 Encore qu'enclin au scepticisme, Kant évite de se prononcer :

Ich weiß nicht, ob jemand an mir eine Spur von einer zum Wunderbaren geneigten Gemütsart oder von einer Schwäche, die leicht zum Glauben bewogen wird, sollte jemals haben wahrnehmen können. So viel ist gewiß, daß ungeachtet aller Geschichten von Erscheinungen und Handlungen des Geisterreichs, davon mir eine große Menge der wahrscheinlichsten bekannt ist, ich doch jederzeit der Regel der 
gesunden Vernunft am gemäßesten zu sein erachtet habe, sich auf die verneinende Seite zu lenken; nicht als ob ich vermeinet, die Unmöglichkeit davon eingesehen zu haben (denn, wie wenig ist uns doch von der Natur eines Geistes bekannt?) sondern, weil sie insgesamt nicht genugsam bewiesen sind; übrigens auch, was die Unbegreiflichkeit dieser Art Erscheinungen, imgleichen ihre Unnützlichkeit anlangt, der Schwierigkeiten so viele sind, dagegen aber des entdeckten Betruges und auch der Leichtigkeit betrogen zu werden, so mancherlei, daß ich, der ich mir überhaupt nicht gerne Ungelegenheiten mache, nicht vor rathsam hielt, mir deswegen auf Kirchhöfen oder in einer Finsternis bange werden zu lassen. Dieses ist die Stellung, in welcher sich mein Gemüt von langer Zeit her befand, bis die Geschichte des Herm Swedenborg mir bekannt gemacht wurde.

Il ajoute que c'est avec impatience qu'il attend la publication du livre de Swedenborg: «Ich warte mit Sehnsucht auf das Buch, das Swedenborg in London herausgeben will». Il le lira dans les années suivantes et portera sur l'auteur et l'ouvrage le jugement que l'on sait.

Le but des Träume eines Geistersehers est beaucoup moins de se prononcer sur l'existence et les manifestations d'«esprits» («Geister»: Kant use rarement du terme «Gespenster»), que de rapprocher les rêves du visionnaire de ceux des métaphysiciens, et de démontrer que les uns et les autres sont également vains, parce qu'ils se targuent de franchir les bornes assignées au savoir humain. La métaphysique, discipline dont Kant se déclare épris encore qu'elle ne lui accorde guère ses faveurs (II, 367), n'est autre que la science des limites de notre raison: «...die Metaphysik [ist] eine Wissenschaft von den Grenzen der menschlichen Vernunft» (II, 368). Il rapporte une remarque attribuée à Aristote, et selon laquelle tous les hommes éveillés ont un monde commun, alors que chaque rêveur a son monde particulier, et ajoute qu'il est disposé à soutenir la converse de cette dernière proposition, à savoir que tout homme vivant dans son monde particulier est un rêveur.

60 Le mot français «visionnaire » - tout comme l'allemand «Visionar» - a sans doute un sens plus vaste et plus vague que celui que Kant donne à «Geisterseher»: conformément à l'étymologie, un «Geisterseher» est un personnage qui voit des esprits. Or, si « esprit » signifie : « être raisonnable », et si l'homme est un être raisonnable, voir des esprits est à la portée du premier venu. - Erreur, répliqueront certains : voir un homme, c'est apercevoir son corps, et non pas son âme qui, elle, est un esprit. Un esprit, c'est un être à la fois immatériel et raisonnable, encore qu'étendu : «Wesen also, welche die Eigenschaft der Undurchdringlichkeit nicht an sich haben, und deren so viele, als man auch will, vereinigt niemals ein solides Ganze ausmachen. Einfache Wesen von dieser Art werden immaterielle Wesen und, wenn sie Vernunft haben, Geister genannt werden» (II, 321). Or, si l'âme humaine est un esprit de cette sorte, où la localiser? Elle sera, diront certains, là où je la sens: dans la tête, le cœur, le diaphragme, les orteils. Mais peut-elle être étendue tout en restant simple, c'est-à-dire indivisible? N'est-il pas plus sage de la localiser dans une glande minuscule, d'où elle gouvernera toute la machine? Vaines spéculations, que nulle expérience n'est en mesure de confirmer ou de réfuter! Au reste, la question même est captieuse, parce qu'elle insinue que l'âme est un être étendu et localisé. «Meine Seele wird also eine einfache Substanz sein. Aber es bleibt durch diesen Beweis noch immer unausgemacht, 
ob sie von der Art derjenigen sei, die in dem Raume vereinigt ein ausgedehntes und undurchdringliches Ganze geben, und also materiell, oder ob sie immateriell und folglich ein Geist sei, ja sogar, ob eine solche Art Wesen als diejenige, so man geistige nennt, nur möglich sei».

(II, 322)

61 Kant, toutefois, se garde d'être dogmatique dans la négation comme dans l'affirmation. Encore que nous ne soyons pas en état de concevoir une entité qui serait à la fois présente et agissante dans l'espace, sans toutefois en remplir une partie comme fait un être matériel, nous devons convenir que la définition donnée d'un «esprit» n'enveloppe pas d'impossibilité, c'est-à-dire de contradiction :

Man kann [...] die Möglichkeit immaterieller Wesen annehmen ohne Besorgniß widerlegt zu werden, wiewohl auch ohne Hoffnung, diese Möglichkeit durch Vernunftgründe beweisen zu können. Solche geistige Naturen würden im Raume gegenwärtig sein, so daß derselbe dem ungeachtet für körperliche Wesen immer durchdringlich bliebe, weil ihre Gegenwart wohl eine Wirksamkeit im Raume, aber nicht dessen Erfüllung d.i. einen Widerstand, als den Grund der Solidität enthielte (II, 323).

62 À l'égard des histoires de revenants fort à la mode en un temps où les "esprits » venaient occuper dans l'imagination du docte comme du vulgaire la place laissée vacante par la disparition des diables et de leurs suppôts, il fait montre de prudence dans une phrase qu'occultistes et parapsychologues se plairont à citer. Il y refuse tout à la fois d'accepter en bloc la masse des histoires qui courent et d'ajouter foi à l'une ou l'autre d'entre elles en particulier, tout en concédant que, à supposer qu'il n'y ait pas de fumée sans feu, il puisse s'en trouver quelque une digne de confiance :

Eben dieselbe Unwissenheit macht auch, daß ich mich nicht unterstehe so gänzlich alle Wahrheit an den mancherlei Geistererzählungen abzuleugnen, doch mit dem gewöhnlichen, obgleich wunderlichen Vorbehalt, eine jede einzelne derselben in Zweifel zu ziehen, allen zusammen genommen, aber einigen Glauben beizumessen. (II, 351)

63 Il expose à nouveau les trois récits dont il avait fait part sept ans plus tôt à Mlle von Knobloch, et sur lesquels son jugement n'a pas varié : attesté par une princesse éclairée et confirmé publiquement par deux ambassadeurs, le premier est assurément digne de confiance, cependant : «Folgende Erzählungen haben keine andere Gewährleistung als die gemeine Sage, deren Beweis sehr mißlich ist» (II, 355). On remarquera que ces trois relations seraient dignes de retenir l'attention des chercheurs auxquels nous donnons aujourd'hui le nom de parapsychologues, parce que les faits seraient attestés par des témoins, et que, à les supposer avérées, les précisions fournies par le voyant ne pourraient être le fait du hasard. En écartant l'hypothèse d'informations fournies par les habitants de l'au-delà, on pourrait estimer que le premier prodige ressortit à la lecture de pensée, et le troisième à la perception extra-sensorielle. Sans donner dans le sophisme que Bergson appellera «illusion rétrospective ", il est permis de penser que Kant avait présent à l'esprit ce que les membres du Cercle de Vienne, qui le rangeront parmi leurs précurseurs, ont appelé "principe de vérification", puis "principe de confirmation»: ne sont recevables que les propositions susceptibles d'être soit confirmées, soit infirmées par l'observation ou le calcul. Si vous m'affirmez qu'en telle contrée vivent des cerbères, c'est-à-dire des chiens tricéphales, je tiendrai assurément votre affirmation pour fausse, mais non pour dépourvue de sens, parce que je sais à quels signes on reconnaîtrait des cerbères, et dans quelles conditions on pourrait établir leur inexistence. Si vous soutenez en revanche que cette contrée est hantée par 
ce que Alfred Ayer appelait des «drogulus ", entendez des êtres invisibles, inaudibles, impalpables, etc., des êtres échappant par nature à toute technique imaginable de détection, mais dont l'existence vous aura été révélée par quelque grâce spéciale ou par un effort de votre réflexion, je tiendrai votre opinion, tout irréfutable qu'elle est, non pas même pour fausse, mais pour irrecevable.

Ce serait une belle chose, disait Kant, si nous pouvions nous former du monde des esprits une conception systématique qui ne fût point déduite de la notion par trop hypothétique de nature spirituelle, mais inférée avec certitude, ou du moins avec vraisemblance, à partir d'une observation sérieuse et universellement acceptée : «Es würde schön sein, wenn eine dergleichen systematische Verfassung der Geisterwelt, als wir sie vorstellen, nicht Jediglich aus dem Begriffe von der geistigen Natur überhaupt, der gar zu sehr hypothetisch ist, sondern aus irgend einer wirklichen und allgemein zugestandenen Beobachtung könnte geschlossen, oder auch nur wahrscheinlich vermuthet werden» (II, 333). Or la plupart des assertions de M. Schwedenberg échappent à tout critère de contrôle. À l'en croire, ses visions seraient de trois sortes : il lui serait arrivé, trois ou quatre fois, entre le sommeil et la veille, de voir, d'entendre, sinon de toucher des esprits; il lui serait arrivé deux ou trois fois d'être transporté en esprit dans un pays éloigné, d'en apercevoir les habitants, les maisons, les forêts, cependant que son corps demeurait à sa place habituelle; il lui arriverait de s'entretenir quotidiennement avec les êtres de l'au-delà, dont les révélations remplissent les in-quarto qu'il livre au public. Encore que leur présence ne s'impose qu'à son sens intime, ils ne laissent pas de lui apparaitre comme s'ils prenaient, hors de lui, figure humaine. Encore qu'ils lui communiquent intuitivement leurs idées, ils paraissent s'exprimer dans la langue usuelle de leur interlocuteur :

Die Gegenwart der Geister trifft zwar nur seinen innern Sinn. Dieses erregt ihm aber die Apparenz derselben als außer ihm und zwar unter einer menschlichen Figur. Die Geistersprache ist eine unmittelbare Mittheilung der Ideen, sie ist aber jederzeit mit der Apparenz derjenigen Sprache verbunden, die er sonst spricht, und wird vorgestellt als außer ihm.

(II, 362)

Chez un sujet normal à l'état de veille, le focus imaginarius, le lieu où apparaît l'objet, se situe en moi dans les jeux de l'imagination, hors de moi dans l'exercice de l'activité sensorielle. Il n'en va autrement que dans les dérèglements de l'esprit, voire dans la folie :

Wenn man dieses einräumt, so dünkt mich, daß ich über diejenige Art von Störung des Gemüths, die man den Wahnsinn und im höhern Grade die Verrückung nenni, etwas Begreifliches zur Ursache anführen könne. Das Eigenthümliche dieser Krankheit besteht darin: daß der verworrene Mensch bloße Gegenstände seiner Einbildung außer sich versetzt und als wirklich vor ihm gegenwärtige Dinge ansieht.

(II, 346)

Il nous est assurément loisible d'avancer des hypothèses, mais à condition qu'elles se prêtent quelque jour au contrôle effectué par des esprits normaux. Ni les spéculations théoriques, ni les visions des illuminés ne sont en mesure d'enrichir notre savoir :

[man kann] nur den Erfahrungen das Recht der Entscheidung einräumen, so wie ich es auch der Zeit, welche Erfahrung bringt, überlasse, etwas über die gepriesene Heilkräfte des Magnets in Zahnkrankheiten auszumachen, wenn sie eben so viel Beobachtungen wird vorzeigen können, daß magnetische Stäbe auf Fleisch und Knochen wirken, als wir schon vor uns haben, daß es auf Eisen und Stahl geschehe. 
Wenn aber gewisse angebliche Erfahrungen sich in kein unter den meisten Menschen einstimmiges Gesetz der Empfindung bringen lassen und also nur eine Regellosigkeit in den Zeugnissen der Sinne beweisen würden (wie es in der That mit den herumgehenden Geistererzählungen bewandt ist), so ist rathsam sie nur abzubrechen; weil der Mangel der Einstimmung und Gleichförmigkeit alsdann der historischen Erkenntniß alle Beweiskraft nimmt und sie untauglich macht, als ein Fundament $\mathrm{zu}$ irgend einem Gesetze der Erfahrung $\mathrm{zu}$ dienen, worüber der Verstand urtheilen könnte.

(II, 371-372)

67 Si Kant a bien admis que la notion de «Geist» n'enveloppe aucune contradiction, s'il a bien concédé que la manifestation de telles entités n'est pas a priori impossible, le passage qu'on vient de lire restreint fort la portée de ces remarques inspirées par la prudence.

Certains se laissent persuader par les arguments métaphysiques en faveur de la survie, et d'autres ajoutent foi aux histoires de revenants. À défaut de s'appuyer sur des preuves convaincantes, leur croyance commune s'enracine dans le désir de survivre. Ecartez cette cause, et les raisons qu'ils invoquent paraîtront ridicules et vaines (II, 349-350). D’autre part, visionnaires et métaphysiciens inversent la relation normale de la connaissance et de l'action. Ce n'est pas parce que nous croyons détenir une preuve de la survie que nous devons nous plier aux règles d'une vie vertueuse; c'est au contraire parce que nous pratiquons la vertu que nous sommes en droit d'espérer une survie bienheureuse.

D'autre part, les connaissances que certains prétendent acquérir de l'autre monde ne peuvent s'obtenir qu'au prix d'une dépense de l'intelligence utile dans celui-ci : «die anschauende Kenntniß der andern Welt [kann] allhier nur erlangt werden, indem man etwas von demjenigen Verstande einbüßt, welchen man für die gegenwärtige nöthig hat» (341). Et Kant conclut son essai en nous invitant, comme le Candide de Voltaire, à renoncer aux vaines controverses pour cultiver notre jardin.

Telles étaient les leçons que le sage de Königsberg livrait au public en 1766, dans la quarante-deuxième année de son âge. Respect du savoir fondé en raison, conscience des limites imposées à l'esprit humain, primauté d'une morale sur laquelle se fonde la croyance en Dieu, en la liberté et en la survie, ces thèmes des Critiques sont esquissés dans les Träume eines Geistersehers.

Les auteurs dont nous allons passer en revue les opinions n'oublieront pas Immanuel Kant. C'est à lui vraisemblablement que Jung-Stilling empruntera l'idée que l'espace et le temps, formes de notre sensibilité, n'ont pas de réalité propre. Il n'en sera que plus à l'aise pour égaler Swedenborg en extravagance. C'est à Kant que Schopenhauer empruntera la distinction du phénomène et de la chose en soi, tout en affirmant que cette dernière est connaissable, qu'elle s'identifie à la Volonté, et qu'elle se manifeste dans des expériences auxquelles Kant n'aurait accordé aucune valeur. Enfin, à la faveur de contresens portant sur le vocabulaire et de citations séparées de leur contexte, Carl du Prel fera de Kant l'homme d'une vision mystique, le génie mal informé qui n'eût pas manqué de se convertir à l'occultisme s'il avait su faire tourner les tables. 
Dans la dernière partie de son livre, Jung-Stilling ${ }^{10}$ résume sa conception du monde des esprits. Certaines créatures de Dieu, les esprits, les anges et les hommes sont dotés de raison et de libre arbitre. Les hommes, qui n'ont qu'une connaissance imparfaite de leur propre nature, n'en ont aucune de celle des autres. Notre connaissance repose en effet sur les données que nous fournissent, dans le cadre de l'espace et du temps, nos cinq sens corporels. Mais ni l'espace ni le temps n'ont de réalité propre, et les êtres qui ne sont pas constitués comme nous ont une autre perception du monde. Toutefois une substance unique, dont la lumière, l'électricité, le magnétisme et l'éther ne sont que des modes, forme le lien qui rattache le monde sensible au monde spirituel. Ce lien se manifeste particulièrement lorsque l'âme humaine, échappant aux servitudes que lui imposent le corps et les sens, entre en rapport avec les esprits. Ces esprits finis, parmi lesquels figurent les âmes humaines séparées de leurs corps, vivent dans le temps, mais non dans l'espace. L'espace n'étant qu'une forme de la représentation, ils se trouvent toujours là où ils pensent être ; mais, différents en cela de la Divinité, ils perçoivent les choses selon l'ordre de la succession. Quel que soit notre désir de savoir, ce serait une faute grave et une erreur funeste que de prétendre accéder, par la pratique des arts magiques par exemple, à des connaissances qui nous sont interdites. Le don de prémonition n'est accordé que parcimonieusement à certains hommes modestes et sages qui ne le recherchent pas. Abusés par les esprits malins qu'ils ne savent pas distinguer des bons, des hommes présomptueux s'exposent aux erreurs les plus funestes.

73 L'éther immense est l'élément où évoluent les esprits bienheureux; l'enveloppe brumeuse de la terre est le séjour des anges déchus et des âmes pécheresses mortes dans l'impénitence. À la fin des temps, l'atmosphère sera purifiée, démons et damnés étant précipités dans un abîme localisé au centre de la terre.

74 Après la mort, les âmes attachées aux désirs charnels demeurent près des corps dans les tombes ; celles des hommes imparfaits encore que non corrompus souffrent quelque temps de la perte de leurs biens terrestres avant de jouir d'un bonheur limité ; celles des vrais chrétiens sont portées par les anges dans les régions de la lumière pure, alors que celles des impies sont précipitées dans les ténèbres.

Les âmes des défunts possèdent des aptitudes créatrices plus grandes que celles dont elles jouissaient lorsqu'elles étaient emprisonnées dans le corps. C'est ce qui leur permet de réaliser ce qu'elles imaginent. Mais cette aptitude a des limites. C'est ainsi que celles qui ne sont pas encore mortes au monde s'efforcent en vain de jouir encore des plaisirs terrestres. Hélas, privées des organes des sens, désormais incapables de goûter aux plaisirs du corps, elles se manifestent sous la forme de fantômes sans consistance qui hantent les lieux de leurs anciens divertissements :

Seelen die der Welt noch nicht abgestorben sind, deren Imagination noch mit lauter Lieblings-Vorstellungen aus ihrem vergangenen Leben angefüllt ist; suchen diese Vorstellungen zu realisiren, aber es sind leere Dunstwesen, die keinen Genuß gewähren können, und eben so wenig ist auch die Seele fähig zum geniesen, sie hat keine sinnlichen Werkzeuge mehr. Daher die bekannten Spuckereyen in alten Gebauden, wo die verarmaten Geister ihre ehemaligen Lustbarkeiten zu wiederholen suchen.

(5. Hauptstück, § 39, 373) 
D'autres, bien que dignes de la félicité, souffrent dans l'au-delà de n'avoir pu réaliser de leur vivant quelque désir. C'est la raison pour laquelle elles apparaissent aux vivants :

Wenn Seelen mit einer unbefriedigten Sehnsucht aus diesem Leben scheiden, so empfinden sie schwere Leiden, wenn sie auch sonst der Seeligkeit fähig sind. Um diese Leiden loß zu werden, sehnen sie sich oft nach einem lebenden Menschen, der jene Sehnsucht befriedigen soll, und wenden die ihnen bekannten Mittel an, zu ihrem Zweck zu gelangen, daher dann die Geistererscheinungen.

(5. Hauptstück, § 41, 374)

77 À quelques exceptions près, ces apparitions sont contraires à l'ordre divin. Comme leurs connaissances sont bornées, les revenants sont capables de se tromper, voire de nous abuser. Il faut donc se garder d'entretenir un commerce avec eux. Toutefois, comme ils ne sont ni des élus ni des damnés, habitants de lieux d'où l'on ne revient pas, mais des êtres qui souffrent, il faut considérer leurs requêtes avec circonspection certes, mais avec attention et bienveillance.

Les expériences magnétiques et les apparitions des esprits apportent la preuve de deux erreurs: celle de la philosophie matérialiste tout comme celle de la croyance au sommeil dans lequel serait plongée l'âme entre la mort et le jour de la résurrection de la chair. Elles démontrent en effet que, non contentes d'exister, les âmes sont en mesure d'agir sans disposer d'un instrument corporel. Mais, à la fin des temps, tou tes les âmes seront réunies à un corps, afin de jouir avec lui des splendeurs du ciel, ou de connaître au centre de la terre les tortures de l'enfer. Et Jung-Stilling conclut son livre par cette invocation : «Dieu de miséricorde, toi qui es l'Amour éternel, épargne à tous les lecteurs de ce livre cet effroyable sort. Amen!».

79 Le livre de Jung-Stilling est avant tout un ouvrage d'apologétique. Les histoires de revenants qu'il rapporte et commente ressortissent au "surnaturel sensible». Elles apportent la preuve expérimentale de la survie des âmes et de la réalité du monde des esprits. L'auteur prétend parler au nom de la Bible et au nom de la raison. Rejetée par les matérialistes, l'autorité de l'Écriture est affaiblie par ceux qui, dédaignant son contenu dogmatique, n'en retiennent qu'un message moral, voire par ceux qui, l'acceptant sans réserve, soutiennent qu'il n'y a plus de prodiges depuis les temps apostoliq ues. La raison est bafouée par les esprits crédules qui, acceptant sans critique toutes les histoires merveilleuses, font le jeu des railleurs et des libertins. Or, l'incrédulité et la superstition relèvent l'une et l'autre d'un esprit de parti qui refuse d'examiner les faits. Parmi la foule des contes, il se trouve quelques récits si bien attestés que la raison oblige d'en reconnaître la vérité. Dûment établies, les apparitions récentes renforcent la crédibilité de celles qui sont rapportées par l'Écriture.

Jung-Stilling ne conteste pas l'existence d'hallucinations chez les malades et les esprits imaginatifs. Pour distinguer une apparition véritable d'une simple vision, il propose deux critères. Le fantôme doit se manifester à l'improviste à plusieurs témoins indépendants et non prévenus; ou à un seul, à qui il révèle quelque secret. Ces règles estimables sont d'une application difficile, et le livre de Jung-Stilling en apporte la preuve. Il fait grand cas d'un prodige dont la relation occupe les dernières pages de son livre (351-360): «Die wichtigste, merkwürdigste, und geheimnisvolleste Erscheinung habe ich aufs Letzte verspart, mit der ich dann auch dies Werk beschliesen will; sie betrift die berühmte weiße Frau» (IV. Hauptstück, $\S 245,351$ ). Les témoignages abondent, certes, mais ils émanent de témoins informés d'une tradition fort répandue. Jung-Stilling rapporte par le menu une autre affaire célèbre (IV. Hauptstück, § 205-208, 
280-288). Feu Georges Villiers, duc de Buckingham, apparut à trois reprises à l'un de ses anciens serviteurs, réputé sobre et pieux, pour qu'il mette en garde son fils menacé d'assassinat. Afin que ce dernier ne prît pas le messager pour un visionnaire, le défunt lui confia des secrets qui n'étaient connus que de son fils et de lui-même. Cette histoire saisissante repose sur un témoignage fragile, celui de Henry Ceeley, un collègue de Parker, à qui ce dernier aurait rapporté la chose. Et Jung-Stilling, qui n'avait décidément pas la main heureuse, s'étend longuement sur la prétendue prophétie de Cazotte (HI, Hau ptstück, § 149-152, 158-176). Chez Cazotte, la piété et le savoir s'allient au don de prophétie. Et ilest moralement impossible que La Harpe, libertin converti sur ses vieux jours, ait inventé l'histoire. La conclusion est saisissante : «Diese Geschichte ist also gewiß und wahrhaftig wahr. - Wenn sie das aber ist, so frage ich jeden vernünftigen und unpartheyischen Menschen, ob es seit der Apostel Zeiten ein merk würtigeres und wichtigeres Zeugniß für die Existenz des Geisterreichs, und dessen Einwürkung auf die sichtbare Weil, gebe?» (III. Hauptstück, § 152, 172). Depuis longtemps, hélas, la Prophétie de Cazotte a rejoint l'Apparition de Mrs Veal et la Vision de Charles XI au musée des mystifications.

81 Toutefois Jung-Stilling donne d'utiles conseils à ceux qui se trouveraient à l'improviste en présence de quelque revenant. Comme le personnage peut être un émissaire du diable, partant un individu dangereux, il faut le sommer, au nom de Jésus-Christ, de préciser son origine et d'expliquer sa présence. S'il s'agit d'une pauvre âme errante qui croit à tort que les vivants peuvent lui être de quelque secours, il est recommandé de lui adresser une homélie dont Jung-Stilling nous donne plusieurs modèles, par exemple :

Gute liebe Seele! du bist auf einem Irrwege; um das Irrdische bekümmere dich nicht weiter, das ist deiner Aufmerksamkeit nicht werth, dafür laß du mich sorgen. Alles! Alles was du noch zu berichtigen hast, wollen wir, deine Freunde, untersuchen, und dann in Ordnung bringen, so daß jedermann mit dir zufrieden werden soll ; und ist noch etwas auf deiner Seelen, das wir nicht gut machen können, so wende dich zu deinem Erlöser, der kann alles berichtigen; zu Ihm! zu Ihm richte nun deine ganze Sehnsucht, da findest du allein Ruhe, der Herr seegne dich! und gebe dir Friede!

(IV. Hauptstück, § 224, S. 316).

Ceci dit, congédiez-le avec bienveillance, en le priant de ne plus se manifester : ich sage dir im Namen Jesu Christi, daß du mich nichl berühren darfst (S. 317).

Deux détails parmi d'autres montrent que, chez Jung-Stilling, le souci d'édification l'emporte sur l'esprit critique. Le premier tend à démontrer que, loin d'être indifférentes à notre sort, les âmes des trépassés s'y intéressent avec sollicitude, l'autre que, pour àccéder à la béatitude dans l'au-delà, il ne suffit pas de supporter avec dignité les souff rances terrestres, mais les surmonter par l'amour. Qui voit son double va mourir. Est-ce le symptôme d'un mal organique profond comme le pensent les médecins? une première escapade du corps astral hors du corps terrestre comme le soutiennent les occultistes? Nullement! Voici pourquoi, selon Jung-Stilling, Mme von M... se vit elle-même avant de mourir : «Nach meiner Theorie war es ein Wesen aus der Geisterwelt, das in seinem irrdischen Leben die Familie von M... oder diese Dame selbst, sehr geliebt, und nun ihren nahen Tod erfahren hatte; die Sehnsucht ihr dieses kund zu 
thun, damit sie sich dazu vorbereiten mochte, bewog es zu dieser Erscheinung» (IV. Hauptstück, § 204, 279). Quant à la Dame blanche, Bertha von Rosenberg, Jung-Stilling estime «daß [sie] mit unversohntem Gemüth und mit bitterm Groil gegen ihren Gemahl gestorben ist, mag wohl die Hauptursache ihres traurigen Umherpilgerns, und ihrer Entfernung vom Anschauen Gottes seyn. Konnte sie den Quell der Liebe in sich erofnen, so ware ihr bald geholfen: denn ihre übrigen Eigenschaften, besonders ihre Wohlthätigkeit, lassen mich hoffen, daß sie dereinst werde zu Gnaden angenommen werden» (IV. Hauptstück, § 254, 358-359).

La métaphysique de Jung-Stilling soulève quelques petites difficultés. Sa conception de l'espace rappelle celle de Kant: «[Es] existirt ausser uns in der Natur kein Raum, sondern unsre Vorstellung desselben, hat ihren Grund allein in unserer Organisation» (V. Hauptstück, § 7, 363). Il en résulte que : «alle endliche Geister [...] leben nicht im Raum » $(15,365)$. «Der Raum ist bloß die Würkung der kürperlichen sinnlichen Werkzeuge, ausser ihnen existirt er gar nicht» $(\S 16,365)$. On s'explique mal comment certaines âmes peuvent demeurer dans le cercueil où est enfermé leur corps, hanter les lieux de leurs plaisirs terrestres, ou apparaitre aux vivants. On comprend plus mal encore que Jung-Stilling ajoute de bonne foi :

Der unermeßliche Aether im Raum unseres Weltsystems, ist das Element der Geister; in dem sie auch leben und weben. Besonders ist der Dunstkreis um unsere Erde bis in den Mittelpunkt derselben, und vorzüglich die Nacht, der Auffenthalt der gefallenen Engel, und solcher Menschenseelen, dit unbekehrt sterben. Diesen ganzen Ramn nennt die Bibel Scheol und Hades, das ist Totenbehälter.

(\$ 30, 370-371)

Je suis enclin à penser que l'espace se venge de ceux qui nient son existence, et qu'il est difficile de concilier les spéculations de la raison et les vérités de la foi.

\section{V}

Schopenhauer se distingue des autres théoriciens des apparitions par ses idées originales, voire plausibles, et leur agencement ingénieux, sinon convaincant. Il a consacré à la question un essai compact d'une centaine de pages publié en 1851 dans ses Parerga und Paralipomena: Versuch über das Geistersehn und was damit zusammenhängt ${ }^{11}$. L'auteur s'appuie sur une documentation considérable, dont témoigne l'abondance des références dans l'ouvrage et la liste des livres de sa bibliothèque personnelle. Encore que négligé par la plupart des philosophes et des occultistes, l'Essai retient toujours l'attention des parapsychologues qui, tout en récusant la métaphysique qu'il prétend illustrer, le considèrent comme un texte classique de leur discipline. Voici d'abord, d'après l'auteur lui-même (161-190), la liste des phénomènes dont il nous propose une interprétation.

Les théoriciens du fantastique, qui ont coutume de grouper les récits autour de thèmes traditionnels, parlent d'histoires de revenants, de doubles, de sorciers, etc. Cette préoccupation est étrangère à la pensée de Schopenhauer, parce que la diversité des apparences l'intéresse moins que la variété des causes. Apparentés par leur aspect, un fantôme suscité par la fièvre et un revenant authentique différent quant à leur nature. D'autre part, selon notre philosophe, toutes les manifestations réellement troublantes ont une origine commune : «Animalischer Magnetismus, sympathetische Kuren, Magie, zweites Gesicht, Wahrträumen, Geistersehen und Visionen aller Art sind verwandte 
Erscheinungen, Zweige eines Stammes...» (146). Schopenhauer distingue cependant neuf degrés parmi les visions, et nous fait accéder, au moyen de cette échelle, des plus banales aux plus prodigieuses.

Les hallucinations provoquées par la fièvre chez un homme normal (a) ou par quelque trouble cérébral chez un dément (b) ont des causes purement organiques. Il en va de même de celles dont peut souffrir accidentellement un sujet sain de corps et d'esprit. Le traitement de ces troubles relève de l'art médical : le philosophe Nicolaï en fut guéri par l'application de sangsues à l'endroit approprié (c). Si ces visions n'ont que des causes, d'autres sont chargées de sens. L'origine de certaines n'est pas trop mystérieuse : informé du mal profond qui le ronge, notre organisme en avertit notre conscience (d). Il est plus difficile d'expliquer l'origine des intersignes qui nous informent d'une menace extérieure : la tradition les attribue à des êtres surnaturels qui, pressentant l'avenir, ont la bonté de nous mettre en garde contre quelque danger, ou de nous inviter à nous préparer à la mort (e). Ces présages sont destinés au visionnaire. Mais il arrive qu'à la faveur de leur don de double vue, certains soient informés, sous une forme symbolique ou manifeste, de la fin imminente d'un voisin ou d'un proche (f). De plus, autant qu'à l'avenir, ce don peut s'appliquer au passé. Tel visionnaire perçoit le fantôme qui hante l'église ou le cimetière, sa demeure ou sa sépulture, l'emplacement de son meurtre ou celui de son trésor. Ce n'est pas que le mort soit présent en personne au lieu qu'il parait hanter: le fantôme n'est qu'une image formée par le cerveau du visionnaire (g). Cependant certaines visions, de mourants en part iculier, sont si nettes et si vives que les sujets se croient en présence d'hommes en chair et en os (h). Restent les prodiges les moins vraisemblables, entendez les apparitions des morts (i). Encore qu'incroyables à première vue, elles sont si bien attestées, dans des termes semblables, par des hommes graves qui ne pouvaient se connaître, qu'il faut se résigner à reconnaître leur réalité.

$89 \mathrm{Au} 6^{\mathrm{e}}$ chapitre du Chien des Baskerville, Sherlock Holmes précise à l'intention de son collaborateur sa conception de la division du travail :

I will not bias your mind by suggesting theories or suspicions, Watson, said he; I wish you simply to report facts in the fullest possible manner to me, and you can leave me to do the theorizing.

Cette répartition des tâches s'impose parfois de manière aussi nette à la théorie des apparitions qu'à l'enquête policière. Schopenhauer, par exemple, entend se confiner dans le rôle de Holmes : «... teils ist mein Fach nicht das erzahlende, also auch nicht die Darlegung von Tatsachen, sondern die Theorie zu denselben, teils müßte ich ein dickes Buch schreiben...» (98). Si convaincu qu'il soit de sa supériorité intellectuelle, le théoricien n'en prend pas moins la défense de l'observateur. Faute de fondations solides faites d'informations précises et de relations sincères, son édifice intellectuel serait fort menacé. Schopenhauer ne met pas en doute la réalité des faits étranges qui passionnaient ses contemporains: «Wer heutzutage die Tatsachen des animalischen Magnetismus und seines Hellsehens bezweifelt, ist nicht unglaubig, sondern unwissend zu nennen» (98). L'auteur ne fera état que de faits bien établis, soit par d'autres, soit par lui-même: «wenn ich etwas als faktisch feststehend annehme, es mir aus guten Quellen, oder aus eigener Erfahrung bekannt sei» (98). Sur ce dernier point, son apport 
est maigre : une de ses servantes eut un rêve prémonitoire (131) ; lui-même devina trois numéros de loterie sur quatre (200). Mais Holmes incite le lecteur à interroger Watson : qu'il ne se contente pas de lire les citations présentées par le philosophe, mais qu'il parcoure quelques-uns au moins des nombreux ouvrages consacrés aux apparitions et au magnétisme animal (98).

91 Toutefois, les exemples qu'il cite et les curiosités qu'il allègue incitent à la circonspection. Peut-on faire état, en faveur de la double vue, du témoignage douteux d'Homère, et de celui, fort suspect, de Cazotte ? (168).

92 Il y a plus grave: Schopenhauer ne marchande pas sa confiance à plusieurs de ses contemporains, Justinus Kerner en particulier. Il estime que les relations qui figurent dans La Voyante de Prevorst et dans les autres écrits de Justinus Kerner comptent parmi les récits d'apparitions («Geistererscheinungen») les plus dignes de foi qui aient été publiés (205). Voici cependant l'une d'elles, dont il fait état dans son essai :

Am 30. März schrieb Herr Pfarrer Herrmann von hier auf ein Blatt nachstehende Fragen und bat sie [die Seherin] diese Fragen an den Geist bei der nächsten Erscheinung zu richten. Sie waren folgende:

Kennst du die Mutter unsers Herrn? Können ihre Fürbitten noch jetzt im Himmel verlangt werden, und sind sie kräftig? Steht sie mit ihrem Sohne in engerer Verbindung als jeder andere Geist?

Nachts 12 Uhr erschien ihr der Geist. Er stand unweit ihres Bettes, als sie von der Wärterin noch Suppe verlangte. Sie aß ruhig ihre Suppe, und erst als sie dieselbe gegessen halte, kam der Geist näher an ihr Bett.

Sie hielt ihm nun, ohne etwas zu sprechen, vor dem Gebet mit ihm, die Handschrift des Herrn $\mathrm{H}$. vor, und er sprach ganz langsarn, so langsam, sagte sie, daß sie geglaubt habe, sie könne das Ende nicht erwarten, folgendes:

Ich kenne die Mutter unsers Herrn etwas besser als du. Für mich bitten kann sie, wie ein jeder selige Geist. Es besteht keine engere Verbindung zwischen ihr und unserm Erlöser - aber - Sie sagte: Er sprach nicht weiter, und als ich wissen wollte, was dies Aber bedeute, sagte er: Es hat eine schöne Bedeutung und ist allen seligen Geistern höchst wichtig - mehr kann ich dir nicht sagen ${ }^{12}$

93 Ce voltairien de Schopenhauer admire la conduite naturelle et familière de la dame envers ses visiteurs d'outre-tombe. Il y voit même une marque d'authenticité, l'absence de crainte étant, à son sentiment, un trait caractéristique des apparitions véritables (159). Mais sa bonne volonté a des limites. Il lui arrive de déplorer la «Leichtgläubigkeit unseres sonst sehr achtungswerten und verdienstvollen Justinus Kerner» (151). S'il admet que Friederike Hauffe, la voyante de Kerner, a cru voir des esprits, ilestime que les discours qu'elle leur prête doivent être «als das Werk ihrer eigenen Einbildungskraft anzusehen» (174), et que, comme les enfants qui s'entretiennent avec un interlocuteur imaginaire, non contente de leur poser des questions, sans s'en rendre compte, elle leur dicte aussi les réponses (175). Il n'aurait pas eu la naïveté de prendre au sérieux les affirmations d'une somnambule prétendant que, dans la langue des esprits, le chien se dit : ni monarto; le chat : na clemos, et c'est parce que je t'aime que je te taquine : clemor tona in diu aswinor ${ }^{13}$. Et, sur un point au moins, il se montre intraitable : que Watson se garde de chasser sur les terres de Holmes. Et de pester contre les interprétations proposées par une «höchst unwissende, gänzlich in ihren Katechismusglauben eingelebte Seherin» $(205)^{14}$.

Dans la conclusion de son essai, Schopenhauer affiche sa satisfaction avec une modestie suspecte. Sa théorie nous éclaire enfin sur la nature de faits aussi intéressants qu'importants, et sur lesquels s'affrontent, depuis des millénaires, le parti de ceux qui 
vont répétant : «es ist», et le parti de ceux qui rabâchent : es kann nicht sein». N'en déplaise aux uns, les faits ne sont pas seulement possibles, mais réels, n'en déplaise aux autres, ils ne sont pas ce qu'on imagine. En voici les grandes lignes.

S'écartant en cela de ses informateurs, Schopenhauer ne croit pas l'espace peuplé d'« esprits", sortes de vertébrés gazeux s'offrant à la vue et à l'ouïe de privilégiés, comme les vertébrés de chair font à la vue et l'ouie du commun des hommes. Les apparitions sont plutôt des hallucinations, mais des hallucinations vraies, perçues par un hypothétique « Traumorgan » - littéralement, un « organe du rêve ». Ce n'est pas un organe au sens étroitement anatomique du mot, parce qu'on en a vainement cherché la trace chez les êtres vivants: «Man hat sich sehr bemüht, dem physiologischen Organ oder dem Sitz dieser Wahrnehmung auf die Spur zu kommen, doch ist es damit bisher noch nicht gelu ngen» (115). Le "Traumorgan » est plutôt une fonction qui utilise en vue de fins particulières, non plus, comme le voulait la tradition, les organes des sens, mais ceux du système nerveux. Schopenhauer l'aurait volontiers appelé « double vue » si l'usage n'avait réservé cette expression pour désigner certaines manifestations particulières, encore que remarquables, d'une fonction générale :

Jene schottische Benennung [das zweite Gesicht] würde [...] die passendste sein, um die ganze Gattung der hierhergehbrigen Phänomene zu bezeichnen und sie auf ein Grundvermögen zurückzuführen; da jedoch die Erfinder derselben sie zur Bezeichnung einer besonderen, seltenen und höchst merkwürdigen Äußerung jenes Vermögens verwendet haben, so darf ich nicht, so gern ich es auch möchte, sie gebrauchen, die ganze Gattung jener Anschauungen, oder genauer, das subjektive Vermögen, welches sich ihnen allen kundgibt, zu bezeichnen. Für dieses bleibt mir daher keine passendere Benennung, als die des Traumorgans, als welche die ganze in Rede stehende Anschauungsweise durch diejenige Äußerung derselben bezeichnet, die jedem bekannt und geläufig ist.

Au début de son essai, Schopenhauer avait en effet exposé sa théorie du rêve. C'est à tort que nous croyons cette expérience banale différente de l'apparition, expérience extraordinaire. C'est à tort aussi que nous l'apparentons à la rêverie. Alors que nous gouvernons cette dernière à notre guise, les images du rêve s'imposent à nous avec la même évidence que les images sensorielles. Qu'elles nous plaisent ou non, elles nous subjuguent. Bien plus, tout comme la perception sensorielle, le rêve permet d'appréhender la vérité : Schopenhauer se plaît à rapprocher «Wahrnehmen» et «Wahrträumen». Or, les connaissances dont nous sommes redevables aux rêves lucides ne sont pas soumises aux limitations qui s'imposent à la perception sensorielle. Les yeux clos dans une chambre obscure, le dormeur peut en percevoir tous les détails, comme si le regard d'un œil intérieur était en mesure de traverser la boîte cranienne et de percer l'obscurité. Capable d'assister à des scènes qui se déroulent en des lieux éloignés, la vision onirique échappe aux limites que l'espace impose à la perception visuelle. Et, pas plus qu'à l'espace, elle n'est soumise au temps : témoin ces rêves prémonitoires dont l'existence n'est pas douteuse. Une servante de Schopenhauer rapporte à sa camarade le rêve qu'elle vient de faire : elle doit réparer les dégâts causés par un encrier renversé. Or l'accident se produira le jour même. Il est également faux de prétendre que tout songe est mensonge et que «Träume sind Schäume». Les rêves montent des profondeurs de la réalité par excellence, à laquelle Schopenhauer donne le nom de Volonté.

Pour expliquer le fonctionnement de son «Traumorgan», Schopenhauer se rallie à l'hypothèse, avancée par Reil, d'une bipolarité du système nerveux, qui s'exprimerait par l'opposition du cerveau et du plexus solaire, ce cerveau abdominal. Quand le 
premier dort, le second veille. Or, mieux encore que le sommeil naturel, le sommeil magnétique, plus profond, favorise la perception extrasensorielle. D'où l'hypothèse selon laquelle le plexus solaire serait le siège du «Traumorgan». La plupart des sujets clairvoyants localisent en effet leur don dans le creux épigastrique («Herzgrube»). Mais se rallier à cette vue serait vraisemblablement assigner un rôle excessif à un organe minuscule au regard de la masse de celui qui remplit la boîte crânienne. Et Schopenhauer est amené à penser que chacun des deux cerveaux joue son rôle dans les phénomènes qu'il étudie.

Nous savons que le système nerveux autonome est en relation avec le système nerveux central. Nous savons aussi que les nerfs sensoriels transmettent au cerveau les excitations captées par leurs récepteurs, et qu'à des causes physiques différentes, un nerf déterminé répond selon sa spécialisation; sensation de couleur pour un nerf visuel, de bruit pour un nerf auditif, etc. lien va de même si le nerf est excité sur un point de son trajet.

Sur ces points, les connaissances de Schopenhauer ne diffèrent guère des nôtres. Mais, ne connaissant pas le rôle que jouent dans la perception les aires corticales de projection, il ignore que les faits psychiques dépendent directement de la phase cérébrale du fait organique. C'est ce qui l'amène à croire que les sensations de couleurs, d'odeurs, de saveurs, etc. ne dépendent que des récepteurs et des nerfs sensoriels. D'autre part, il ne dispose, sur ce que nous appelons influx nerveux, que de notions sommaires et inexactes. Il imagine des vibrations se propageant le long du nerf indifféremment dans un sens ou dans l'autre. Il compare ces nerfs à des boules métalliques creuses qu'on peut chauffer de l'intérieur aussi bien que de l'extérieur : «... die Sinnesnerven [...] ktinnen sowohl von ihrem inneren als von ihrem äulleren Ende aus in Tätigkeit versetzt werden; - etwa wie eine eiserne ohlkugel sowohl von innen als von außen glühend gemacht werden kann» (127).

Ce fondement posé, Schopenhauer est en mesure d'expliquer l'origine des visions : la fonction onirique et la fonction sensorielle disposent des mêmes organes corporels, mais elles les utilisent dans des sens opposés : «Das Traumorgan ist [...] dasselbe mit dem Organ des wachen Bewußtseins und Anschauens der Außenwelt, nur gleichsam vom andern Ende angefaßt und in umgekehrter Ordnung gebraucht...» (127). Supposons qu'une excitation issue de l'intérieur de notre organisme gagne le cerveau. Celui-ci va fonctionner à rebours, mais, conformément à son rôle naturel, il imprimera aux excitations sa marque propre, il en tirera des images qui se juxtaposent dans l'espace et se succèdent dans le temps. L'excitation gagne alors les nerfs sensoriels qui se comportent comme le cerveau : ils fonctionnent à rebours, mais réagissant comme s'ils étaient stimulés de l'extérieur, confèrent aux images cérébrales la vivacité qui caractérise les images sensorielles:

Besonders [...] läßt, durch unsere Hypothese, jene so merkwürdige Lebendigkeit der Traumanschauung, jene oben geschilderte, scheinbare Wirklichkeit und Leibhaftigkeit aller im Traume wahrgenommenen Gegenstände sich begreiflich machen, nämlich daraus, daß die aus dem Innern des Organismus kommende und vom Zentrum ausgehende Anregung der Gehirntätigkeit, welche eine der gewähnlichen Richtung entgegengesetzte befolgt, endlich ganz durchdringt, also zuletzt sich bis auf die Nerven der Sinnesorgane erstreckt, welche nunmehr von innen, wie sonst von außen, erregt, in wirkliche Tätigkeit geraten. Demnach haben wir im Traume wirklich Licht-, Farben-, Schall-, Geruchs- und Geschmacksempfindungen, nur ohne die sonst sie erregenden äußeren Ursachen 
bloß vermöge innerer Anregung und infolge einer Einwirkung in umgekehrter Richt ung und umgekehrter Zeitordnung.

101 qu'elle est en soi.

Schopenhauer croit trouver une confirmation de cette hypothèse d'une inversion du courant nerveux dans l'impression de désorientation qui nous surprend parfois au réveil, et nous fait prendre la droite pour la gauche, l'avant pour l'arrière. D'autre part, c'est parce qu'il fait jouer les nerfs sensoriels, que le rêve produit des images plus vives que la rêverie, phénomène purement cérébral (126-127).

Lorsque les deux fonctions utilisent à tour de rôle le même appareil, l'une à l'état de veille, l'autre pendant le sommeil, leurs images n'entrent pas en conflit. C'est la raison pour laquelle il arrive que des visions s'imposent avec une évidence telle que le sujet les prend pour des réalités du monde matériel. Mais il n'en est pas toujours ainsi : il arrive que les visions d'origine interne soient faiblement colorées, grises, blanchâtres, quasi transparentes. Les voix n'émettent que des sons faibles ou rauques, des chuchotements, des pépiements. Lorsqu'on porte l'attention sur elles, les visions s'évanouissent, ou s'estompent. Elles cherchent refuge dans un coin sombre, derrière un rideau devenu transparent. Chacun sait que les fantômes affectionnent le silence et l'obscurité, et qu'ils évitent les lieux éclairés et les sociétés bruyantes :

Wenn der Seher [der Gestalten] eine geschärfte Aufmerksamkeit auf sie richtet, pflegen sie zu verschwinden, weil sie dem äußeren Eindruck sich jetzt mit Anstrengung zuwendenden Sinne nun diesen wirklich empfangen, der, als der stärkere und in entgegengesetzter Richtung geschehend, jene ganze,von innen kommende Gehirntätigkeit überwältigt und zurückdrängt. Eben um diese Kollision zu vermeiden geschieht es, daß, bei Visionen, das innere Auge die Gestalten soviel wie möglich dahin projiziert, wo das äußere nichts sieht, in finstere Winkel, hinter Vorhänge, die plötzlich durchsichtig werden, und überhaupt in die Dunkelheit der Nacht, als welche bloß darum die Geisterzeit ist... (158)

Si le mécanisme imaginé par Schopenhauer est fort capable d'expliquer des phénomènes curieux, certes, mais réputés naturels, comme les songes et les hallucinations, il n'est pas encore en mesure de rendre compte de faits plus troublants, comme les rêves prémonitoires et les apparitions des mourants à leurs proches. Pour y parvenir, il faut nous aventurer dans le domaine de la métaphysique.

Schopenhauer s'est donné pour le disciple et le continuateur de Kant. Or ce dernier distingue nettement la chose telle qu'elle s'offre à notre connaissance et la chose telle

5 Notre sensibilité - ou plutôt « sensorialité » = «Sinnlichkeit» - dispose de deux cadres qui nous permettent de percevoir les objets comme étendus : l'espace, et successifs : le temps. Complétant notre sensibilité, l'entendement («Verstand») se donne des outils qui lui permettent d'agencer notre savoir: ce sont les catégories, dont la plus importante est vraisemblablement celle de causalité. Il nous est certes permis de penser la chose en soi, mais il ne nous est pas loisible de la connaitre. Elle est assurément étrangère à l'espace et au temps, grâce auxquels se manifestent les apparences, et à la causalité, qui les lie.

Notre philosophe accepte, mais en la modifiant sensiblement, cette théorie de son maître. Le monde, à son sens, présente deux aspects: il est Représentation («Vorstellung») et Volonté («Wille»). Ces deux notions correspondent à celles de phénomène et de chose en soi.

7 La Représentation est un produit de notre cerveau, qui lui impose ces cadres que sont l'espace et le temps, et le soumet aux lois de la causalité. L'espace et le temps 
constituent aussi le principe d'individuation : c'est par eux qu'existent - ou paraissent exister - des êtres distincts, des individus. Issue du cerveau, la Représentation est vouée à disparaître avec lui, tout comme le temps, l'espace et l'individualité.

N'en déplaise à Kant, la chose en soi est connaissable. C'est cette Volonté qui pousse l'insecte sur le point de mourir à déposer en lieu sûr les œufs qui écloront au printemps. C'est elle qui incite les espèces menacées à produire davantage de rejetons. C'est par elle aussi que s'exerce l'action à distance des magnétiseurs. Indépendante de l'espace et du temps, son action a un caractère magique. Accessible à l'intuition du métaphysicien, elle échappe par nature à la vérification du savant.

Justinus Kerner avait intitulé la seconde partie de sa Seherin: «Eröffnungen über das Hereinragen einer Geisterwelt in die unsere», Schopenhauer aurait pu intituler son essai : Über das Hereinragen der Welt als Witte in die Welt als Vorstellung. Présente dans notre organisme, qui est l'une de ses objectivations, et particulièrement dans le système ganglionnaire, la Volonté exerce aussi son action sur le cerveau. Or celui-ci, conformément à sa nature, fait apparaître dans l'espace et le temps des choses qui leur sont par nature étrangères. De là vient que les voyantes croient discerner dans l'espace extérieur des apparences produites par leur cerveau. On ne s'étonnera ni de la vision à distance, ni des prémonitions, ni du caractère contagieux des visions, si l'on se rappelle que la Volonté est par nature étrangère à l'espace, au temps et à l'individualité. Kant avait donné des «Geister» une définition que Schopenhauer qualifie de spiritualiste: «Wesen [...] welche die Eigenschaft der Undurchdringlichkeit nicht an sich haben, und deren so viele als ma $\mathrm{n}$ auch will, vereinigt, niemals ein solides Ganze ausmachen. Einfache Wesen von dieser Art werden immaterielle Wesen und, wenn sie Vernunft haben, Geister genannt werden». Schopenhauer lui oppose sa conception idéaliste : il n'existe point, dans ce monde ou dans un autre, des entités autonomes auxquelles conviendrait le nom d'« esprits » : il n'y a que des images formées par les sujets. Si certaines présentent un caractère « surnaturel ", c'est parce qu'elles sont suscitées par une Volonté omniprésente.

Nombreux sont ceux qui assurent avoir vu le fantôme d'un vivant; mais rares en revanche ceux qui affirment avoir reçu la visitation d'un mort: «Jedenfalls ist eine Geistererscheinung zunächst und unmittelbar nichts weiter, als eine Vision im Gehirn des Geistersehers: dafl von außen ein Sterbender solche erregen konne, hat häufige Erfahrung bezeugt; daß ein Lebender es konne, ist ebenfalls, in mehreren Fällen, von guter Hand beglaubigt worden; die Frage ist blofl, ob auch ein Gestorbener es könne» (205). Car, si la Volonté se manifeste naturellement chez le vivant - fût-il mourant - qui est un sujet, elle le peut difficilement chez le mort, qui a perdu son individualité. L'apparition d'un mort suppose, tant du côté du défunt que du vivant qui l'aperçoit, des conditions difficiles à remplir: "[Dies] wären, bei der Annahme einer wirklichen Geistererscheinung, die auf der Seite des sie wahrnehmenden Subjekts liegenden Schwierigkeiten. Andere wieder liegen auf der Seite des angenommenermaßen einwirkenden Verstorbenen» (202-203). «Da wir das im Tode unversehrt gebliebene innere Wesen des Menschen uns zu denken haben als aufler der Zeit und dem Raume existierend, so konnte eine Einwirk ung desselben auf uns Lebende nur unter sehr vielen Vermittlungen, die alle auf unserer Seite lägen, stattfinden, so daß schwer auszumachen sein würde, wieviel davon wirklich von dem Verstorbenen ausgegangen wäre» (201). La chose n'est cependant pas impossible parce que, si la mort met fin à l'existence individuelle, elle n'affecte en rien la Volonté, qui est éternelle et unique. En 
tant que telle, elle n'agit pas seulement sur l'organisme humain, mais aussi «auf leblose, also unorganische Körper, die demnach durch sie bewegt werden können» (204). C'est ainsi que les manifestations des «Poltergeister» viennent illustrer la métaphysique de Schopenhauer.

L'essai de Schopenhauer ne ressortit ni à l'apologétique comme les ouvrages de Glanvill et de Jung-Stilling, ni à la science comme les travaux de Dessoir ou de Bender. C'est avant tout pour défendre et illustrer sa propre philosophie qu'il l'a composé. Comme il ne se donne pas pour un observateur, mais un théoricien, il ne marchande pas sa confiance aux compilateurs de récits prodigieux. C'est ce qui l'amène à oublier qu'entre la relation et la théorie, il faut accorder une place très large à la critique des témoignages. Or cette dernière est presque aussi ancienne que la croyance aux esprits. Schopenhauer ne pouvait l'ignorer. Mais, s'il s'est refusé à la pratiquer, ce n'est pas seulement parce qu'elle est une tâche modeste et fastidieuse, c'est parce qu'il lui importait assez peu de démêler le bon grain et l'ivraie, l'illusion et la réalité. Son opposition de la Représentation et de la Volonté ne coïncide pas avec l'opposition vulgaire du naturel et du surnaturel. Car la Volonté est présente dans les rencontres les plus banales comme dans les plus extraordinaires. Elle nous accorde, à la faveur d'un même "Traumorgan", nos rêves quotidiens et des songes prophétiques. Elle guide chaque jour vers sa femelle l'insecte minuscule appartenant à l'espèce la plus rare; il la trouvera infailliblement, après avoir parcouru une distance invraisemblable, et saura la distinguer parmi les représentantes des espèces les plus voisines. Et la même Volonté attentive à la conservation des espèces accorde aussi aux individus des connaissances inaccessibles à leur intelligence.

De la théorie de Schopenhauer, les parapsychologues ont surtout retenu que les visions les plus authentiques sont subjectives. À cela près, il en est peut-être de son système comme de beaucoup d'autres, horloges dont les connaisseurs admirent le mécanisme, mais auxquelles ils ne songent guère à demander l'heure.

\section{VI}

Les manifestations du « corps astral » ou du « double », qui passionnent depuis toujours les philosophes et le vulgaire, attirent depuis quelques décennies l'attention des psychologues et des psychiatres. C'est pourquoi je me propose d'examiner deux opinions adverses: celle que l'occultiste Carl du Prel défend dans sa Monistische Seelenlehre. Ein Beitrag zur Lösung des Menschenrätsels (Leipzig, 1888), et celle que E. Menninger-Lerchenthal expose dans un ouvrage médical: Das Truggebilde der eigenen Gestalt. Heautoskopie, Doppelgänger (Berlin, 1935), que complète un livre intitulé : Der eigene Doppelgänger (Bern, 1946).

114 Les deux auteurs ne s'entendent ni sur la nature du phénomène, ni même sur le sens exact du terme qui le désigne. Pour le psychologue, voir son double, c'est être victime d'une hallucination. La pensée qu'un observateur étranger puisse apercevoir cette image n'effleure pas son esprit. Pour l'occultiste, le double est la manifestation du corps astral, réalité objective faite de matière subtile offerte au regard de tous. Les termes de «Doppelgänger» et de "Gespenst» désignent la même entité. Persuadé que la vision du double est subjective, le psychologue s'ef force d'analyser l'expérience vécue du sujet qui l'éprouve, et de découvrir le mécanisme psycho-physiologique qui la déclenche. Convaincu au contraire que le « corps astral » est une réalité objective, localisée dans 
l'espace, l'occultiste fait appel à l'argument d'autorité et pratique, ou se targue de pratiquer, la critique des témoignages. Un point cependant reste obscur à ses yeux : celui du processus à la faveur duquel le corps éthéré se détache du corps charnel : «In Bezug auf den Trennungsprozeß des Astralleibes vom Kürper in der Doppelgângerei sind wir noch vollstä.ndig im Dunklen und müssen die Tatsachen eben hinnehmen, wie sie sind» (Die monistische Seelenlehre, 171). À quoi Menninger-Lerchenthal réplique dans son premier ouvrage par une «neurologisch-psychologische Auffassung der Doppelgangererscheinung» capable de rendre compte du prétendu «Trennungsprozeß» qui intrigue du Prel (Der eigene Doppelgänger, Vorwort).

Menninger-Lerchenthal rend hommage au savoir de l'homme dont il combat les idées : «In dem vor sechzig Jahren erschienenen Buche du Prels: Die monistische Seelenlehre ist alles, was bis dahin und bis jetzt über die Erscheinung des Doppelgängers bekannt war, enthalten» (Der eigene Doppelgänger, S. 52). C'est peut-être faire trop d'honneur à un homme fort savant sans doute et qui, sans être comme beaucoup de ses collègues un esprit vulgaire, n'en était pas moins un esprit faux, parfois tendancieux dans l'interprétation des philosophies, souvent léger dans l'acceptation des preuves.

116 La pensée moderne, cartésienne surtout, fait de l'homme un être double, composé d'un corps physique et d'une âme spirituelle si différents en nature que le problème de leur union et de leur interaction est quasi insoluble. À première vue, du Prel, qui dote l'homme d'un corps astral, en fait un être triple. Il n'en est rien cependant, et lui-même qualifie sa conception de «moniste ». Voici son argumentation : l'âme, telle qu'on la conçoit généralement, n'est qu'une conscience, un «Bewußtsein». Or, la définir de cette sorte, c'est l'identifier à l'une de ses fonctions et négliger l'autre. Autant que connaissante, l'âme est organisatrice. C'est elle qui préside à la formation du corps physique, c'est elle aussi qui modèle le corps astral. Ainsi, la notion centrale de la philosophie de du Prel n'est pas tant celle de corps astral que celle d'âme. Séparée du corps charnel par la mort, notre âme ne laisse pas de subsister, et d'habiter un corps éthéré, comme en témoignent les apparitions des morts.

117 À l'appui de sa thèse, notre occultiste invoque l'autorité d'Aristote, en qui il voit un «Vertreter der monistischen Seelenlehre» (Ibid., 114). On peut concéder que du Prel est fidèle à l'esprit, sinon à la lettre du traité d'Aristote sur l'âme. Celui-ci soutient en fait que l'homme possède non pas une, mais plusieurs âmes subordonnées les unes aux

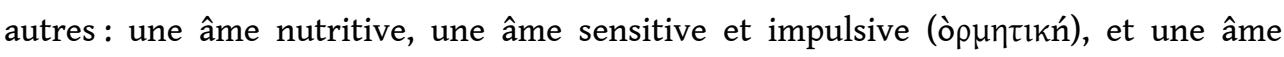

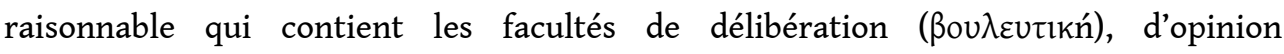

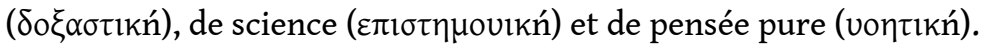

118 La première est commune à tous les êtres vivants, la seconde appartient à l'animal et à l'homme, et la troisième à l'homme seul. Elles jouissent donc d'une sorte d'existence particulière, à cela près que la troisième ne saurait exister sans la seconde, ni la seconde sans la première. Il en résulte, selon Aristote, qu'on ne saurait donner une définition unique de l'âme. Par ailleurs, le Stagirite soutient que l'âme raisonnable survit au corps physique, sans pour autant rester liée, comme chez des philosophes ultérieurs, à quelque corps subtil qui lui servirait de véhicule. À cela près, on peut concéder que les diverses âmes sont en fait des facultés appartenant à une même 
entité, et que celle-ci est étroitement liée au corps. Ainsi serait justifié l'emploi de l'adjectif «moniste ». En fait, plus qu'à la métaphysique, la théorie aristotélicienne de l'âme ressortit à la biologie, et le dualisme cartésien lui est parfaitement étranger.

Quelle que soit sa sympathie pour Aristote, à qui il consacre un chapitre de sa Monistische Seelenlehre, du Prel se donne surtout pour le continuateur de Kant, sur qui il a composé un essai intitulé «Kants mystische Weltanschauung» (Carl du Prels Ausgewählte Schriften, I. Bd). Il y soutient cette thèse que Kant était un mystique à qui malheureusement les connaissances indispensables ont fait défaut.

Ayant cité quelques passages des Träume eines Geistersehers ainsi que de la Psychologie de Kant, puis reproduit en regard quelques développements empruntés à Swedenborg, du Prelconclut sereinement à l'accord de ces deux auteurs, les pressentiments du critique méfiant et les intuitions du visionnaire intrépide se rejoignant dans une synthèse supérieure dont nous sommes redevables aux facultés des sujets hypnotisés, des somnambules et des médiums :

Es geschieht demnach in vollständiger Übereinstimmung mit Kant und Swedenborg, daß ich in meinen mystischen Schriften die Gleichzeitigkeit eines transcendentalen Subjekts mit dem irdischen Menschen ausgesprochen habe, indem ich zunächst aus der Thatsache der dramatischen Spaltung des Ich im Traume die psychologische Möglichkeit, sodann aber aus den Fähigkeiten der Hypnotisierten, Somnambulen und Medien die metaphysische Wirklichkeit dieser Gleichzeitigkeit bewies. Bei Kant, weil er diese Thatsachen nicht kannte, ist seine Erkenntnis rein intuitiv und um so bewundernswerter; Swedenborg dagegen leitete diese Gleichzeitigkeit als logische Folgerung, aus seinem eigenen inneren Leben ab. (Kants mystische Weltanschauung, XXXVI)

121 À supposer qu'il ait eu le bonheur de vivre à la fin du XIX siècle, Kant n'aurait pas manqué d'adhérer, comme Carl du Prel, au spiritisme, et de considérer, tout comme lui, les facultés supranormales de Swedenborg dont font état la lettre à $\mathrm{M}^{\text {lle }}$ von Knobloch et les Träume eines Geistersehers, comme beaucoup mieux établies qu'elles ne le furent à l'âge de l'Auf klarung :

Man muß sehr viel überflüssige Zeit haben, um die Frage zu untersuchen, ob Kant Spiritist war. Wenn aber die Frage gestellt wäre, ob er heute Spiritist sein würde, so müsste ich diese Frage bejahen. Zunächst würde sich ihm der Fall Swedenborg heute günstiger darstellen, als damais. Heute sind die von Kant angeführten Falle von der Sehergabe Swedenborgs viel besser beglaubigt als damals.

(Ibid., LIII)

De l'étude des travaux qui jalonnent la vie de Kant, il ressort «daß Kant sein ganzes Leben hindurch zu mystischen Anschauungen geneigt hat, wobei ihm allerdings der Mangel empirischen Thatsachenmaterials sehr hinderlich war...» (Ibid., XVI).

Comme il me semble pas que du Prel ait chargé quelque médium d'évoquer l'esprit de Kant afin d'en obtenir la confirmation de sa propre interprétation, nous en sommes réduits à prendre acte des déclarations que fit de son vivant le philosophe à Reinhold Bernhard Jachmann, qui fut, de 1784 à 1794 son «amanuensis» et son confident :

Will man [...] nicht mit Worten streiten, will man den Kantischen Ausdrücken, z.B. praktische Vernunft, Vernunftglaube, moralische Schriftdeutung u. a. m. nicht absichtlich einen andern Sinn unterlegen, als der Verfasser sich dabei dachte und 
das aus Gefühlen herleiten, was er einzig und allein auf Vernunft gründete, so wird man auch weder in den Schriften noch in dem Leben Kants irgend etwas Mystisches entdecken. Kant hat sich hierüber auch gegen mich ganz unverhohlen erklärt und versichert, daß keines seiner Worte mystisch gedeutet werden müsse, daß er nie einen rnystischen Sinn damit verbinde und daß er nichts weniger als ein Freund mystischer Gefühle sei. Bei der Gelegenheit tadelte er noch den Hang Hippels zur Mystik und erklärte überhaupt jede Neigung zur mystischen Schwärmerei für eine Folge und für ein Zeichen einer gewissen Verstandesschwäche.

(Immanuel Kant in Briefen an einen Freund, Elfter Brief. Cet ouvrage fut publié en 1804, l'année même de la mort du philosophe, comme second volume d'une série : Über Immanuel Kant)

«Transcendant » et «transcendental» sont deux termes du vocabulaire kantien dont $\mathrm{du}$ Prel fait un usage discret pour le premier, fréquent pour le second. À son sens la psychologie comporterait trois degrés : empirique quand elle se fonde sur les données banales fournies par les sens et l'introspection, transcendentale quand elle traite des facultés cachées qne révèlent le somnambulisme et le magnétisme, transcendante quand, à la faveur du spiritisme, elle a pour objet la nature de l'âme dans l'au-delà. La psychologie transcendentale est le chaînon qui relie l'empirique à la transcendante :

[Es hat keinen Sinn], den Spiritismus isolirt zu studiren, wie es meistens geschieht. Durch den Spiritismus allein gelangen wir noch zu keiner genügenden Definition des künftigen Lebens; ja er bringt uns zu falschen Vorstellungen darüber, wenn wir nicht bedenken, daß das Einwirken der Phantome in die materielle Welt, der sie nicht mehr angepasst sind, gegen ihre Natur ist, ihr eigentliches Leben aber von ganz anderer Art sein muß. Dieses Bedenken gilt auch von der Magie Lebender, und auch diese reicht nicht aus für eine Definition des Jenseits. Erst wenn wir die Phänomenenreihe beider Gebiete verbinden, ergänzen sie sich und werfen gegenseitig Licht auf einander, und wer gar experimentiren will, muß beide kennen. Die transscendentale Psychologie und die transscendente ergänzen sich, und nur Gradunterschiede können zwischen beiden vorhanden sein (Der Tod, das Jenseits, das Leben im Jenseils, München, Verlag des Verfassers, 1899, 78-79. - Souligné par moi). Das unbewußte Leben des Diesseits ist das bewußte Leben des Jenseits. Diese Vermuthung Kant's aber, daß wir schon im Diesseits Geister seien, wenn wir es auch cerebral nicht wissen, und daß die Fähigkeiten der Geister identisch sind mit den magischen Fähigkeiten Lebender, ist nun experimentell erwiesen, indem sowohl die citirten Doppelgänger Lebender, als die Phantome Verstorbener sich in identischer Weise durch schreibende oder sprechende Medien kundgeben können. Daß die transscendente Psychologie in der Thal nur die Fort-setzung der transscendentalen ist, geht auch daraus hervor, daß beide auf die gleiche Weise geweckt werden können.

$$
\text { (Ibid., 87. - souligné par moi) }
$$

Las! si la pensée de du Prel flotte dans la transcendance, son vocabulaire patauge dans le contresens. Kant qualifie en effet de transcendental ce qui rend possible l'application des principes a priori à l'expérience, voire, plus généralement, ce que présuppose toute expérience possible, et de transcendant ce qui n'est objet d'aucune connaissance. L'expérience présuppose en effet des conditions psychologiques et des conditions logiques : «Transzendental-psychologisch sind die Funktionen, welche der Erzeugung der Erfahrung zugrunde liegen, mögen sie nun von der Sinnlichkeit, der Einbildungskraft oder dem Verstande ausgehen; transzendental-logisch ist die Gesetzlichkeit des reinen Denkens, sofern sie unabhangig von der Erfahrung und doch für diese selbst, als allgemein-notwendige Bedingung derselben gilt (R. Eisler, KantLexikon, s.v. «Transzendental»). Et Kant précise dans son Nachlaß: «Transzendentale Begriffe können sinnenfreie genannt werden. Transzendentalphilosophie auch sinnenfreie. Alles worin nich ts Empirisches, aber doch Allgemeingeltendes ist, z.E. die 
Kategorien. Transzendent: was gar kein Erkenntnisstück ist» (4643). Du Prel donne dans le délire d'interprétation. son corps matériel, l'homme dispose d'un corps astral, ainsi nommé parce qu'il est formé de la même matière subtile dont, aux sentiments des Anciens, étaient composés les corps célestes. Le sentiment de l'intégrité de notre corps charnel nous offre un premier argument en faveur de son existence. Différents en cela des êtres inférieurs qui jouissent de la capacité de reconstituer leurs corps mutilés, les animaux supérieurs, et l'homme en particulier, continuent à éprouver l'existence de leurs membres amputés : quand leur corps charnel est mutilé, leur corps astral est intact. Au reste ce corps peut se manif ester aussi bien aux yeux du sujet, en qualité de «Doppelganger», qu'à ceux de témoins étrangers, comme «Gespenst». De ces deux sortes de manifestations, la seconde est assurément la plus digne d'intérêt, parce qu'elle échappe au soupçon de phénomène subjectif et hallucinatoire qui pèse sur la première. À ceux qui font valoir que les fantômes se manifestent dans des conditions pathologiques, il est aisé de répondre qu'une chose ne laisse pas de jouir d'existence propre quand elle est suscitée à la faveur de circonstances anormales. Encore que le mécanisme qui permet au corps astral de se séparer du corps physique nous reste inconnu, nous savons, par des témoignages nombreux que, loin d'être image inconsistante, le premier déploie une activité propre. Au reste, une solidarité étroite lie les deux organismes : toute blessure infligée au corps astral est ressentie par le corps matériel. Encore que nous en connaissions surtout les manifestations spontanées, le corps astral est en mesure de se détacher du corps physique à la faveur de techniques tant traditionnelles que modernes. Alors que l'organisme reste plongé dans un sommeil léthargique, le corps astral prend son essor puis rapporte au retour de son voyàge aussi rapide que gratuit, des précisions sur les spectacles dont il fut témoin. Bien qu'il ait renoncé, en faveur du dualisme, à la croyance qui faisait de l'homme un être triple, le christianisme n'en continue pas moins à professer que les âmes seront unies dans l'au-delà à des corps glorieux. Les apparitions des fantômes en apportent la preuve sensible. (Et ce qu'ils nous apprennent de leur mode de vie nous incite à souhaiter la mort plus qu'à la redouter !)

Du Prel note à juste titre que, s'il est aisé de déduire la réalité du corps astral de principes métaphysiques, les preuves expérimentales de son existence ont un autre pouvoir de persuasion. Et son livre en offre à foison. Certaines ne manquent pas de piquant, comme l'histoire de la femme adultère qui délègue son double auprès du mari pour implorer son pardon (206), ou celle du meurtrier dénoncé par son double, mais relaxé par un juge «in deren Gesetzbüchern das Kapitel der transcendentalpsychologischen Phänomene keinen Raum gefunden» (215). Seule fait défaut la critique des témoignages. Il est vraisemblable que, différent en cela des parapsychologues, notre occultiste estime qu'une foule de témoignages douteux vaut largement un petit nombre de faits bien établis.

Il nous rapporte par exemple l'aventure d'Hermotime de Clazomène, dont le corps astral avait l'habitude d'effectuer des voyages aériens, qui lui permettaient de faire des 
observations curieuses, jusqu'au jour où il eut la surprise de constater que ses ennemis avaient profité de son absence pour détruire son corps physique (265). Il oublie d'ajouter que Pline rapporte l'anecdote sans s'en porter garant (Naturalis historiae, 1. VII, LIII, 174), et que les historiens tiennent le personnage pour légendaire.

Il fait état d'une expérience qu'il juge instructive, encore qu'elle ne soit pas «salonfähig»:

Ein gewisser Meinecke, an Diarrhöe leidend, halte den Drang, den Abort aufzusuchen, wurde jedoch durch einen anwesenden Besuch daran verhindert. Heftig wünschte er die Entfernung des Gastes, und seine Gedanken antizipierten beständig den beabsichtigten Gang. Als endlich der Besuch sich entfernt hatte, eilte Meinecke nach dem Gemache, öffnete die Thüre und sah sich nun selber in den Kleidern, die er eben trug, sitzen.

(177)

130 Alors que du Prel juge le comportement du double « irrationnel », entendez inutile, un psychologue aurait tenu l'expérience pour vraisemblable et digne d'intérêt, parce que tendant à prouver que la réalisation d'un désir pouvait revêtir un caractère «esthésique ».

Il se trouve que le corps astral ne porte pas toujours les mêmes vêtements que le corps charnel. C'est ainsi qu'un sieur Peter Müller s'était fait conduire à l'office religieux par son valet qui rejoignit aussitôt l'écurie. À sa vive surprise, ce dernier aperçut son maitre en robe de chambre et pantoufles, la tête coiffée d'un bonnet blanc, comme ilavait l'habitude d'être vêtu pendant la matinée, allant et venant dans l'écurie. Au domestique troublé, Herr Müller confessa qu'au moment de l'apparition sa pensée s'était détachée de ses devoirs religieux pour se tourner vers son bétail (178).

L'anecdote ne paraît pas invraisemblable, d'une part parce que le valet a pu avoir l'illusion d'apercevoir son maître vêtu comme il l'était d'habitude dans son écurie, et d'autre part parce que les pensées profanes qui occupent l'esprit des fidèles pendant les offices sont si fréquentes qu'elles ne prouvent rien. Comme les illusions et les hallucinations sont des faits avérés, il n'est pas étonnant que les sujets qui en sont victimes aperçoivent, couverte de ses vêtements habituels, leur image ou celle d'une personne étrangère, vivante ou morte. Mais la chose est fo1t embarrassante si l'on tient l'apparition pour une réalité objective. Max Dessoir le note sèchement :

Ein sofort einleuchtendes Bedenken liegt in der Tatsache, daß die Erscheinungen mit Rock und Weste, Stiefeln und Hut bekleidet sind. Selbst wer an einen Astralleib und dessen gelegentliches Sichtbarwerden glaubt, wird doch nicht behaupten, daß es Astral-Wolle und Astral-Leder gibt. Das wäre wirklich krasser Aberglaube (Das Ich, der Tod, der Traum, 1947, 23). - Un auteur aussi crédule que Flammarion «bemerkt dazu, man känne sich schwer vorstellen, daß nicht nur der menschliche Leib, sondern auch seine Bekleidung verdoppelt werde. In der Tat: wer da glaubt, es handle sich um mehr als ein subjektives Bild, der muß auch die Tatsächlichkeit von Ätherblusen und Astralstiefeln zugeben». (Ibid., 174)

Du Prel aurait sans doute jugé l'argument trop vulgaire pour être digne de retenir son attention. Il ne s'est pas demandé, comme d'autres, de quelle matière subtile étaient faits le costume «hechtgrau» que portait le double de Goethe et le cheval qu'il montait. Mais il rapporte gravement, tout comme Flammarion, l'aventure d'Emilie Sagée, dont les élèves apercevaient le double pendant qu'elle enseignait et prenait ses repas. Ce double, assurément couvert de vêtements corrects, ne tenait ni bâton de craie ni fourchette, ce qui laisse entendre que, différents en cela des tissus, ces accessoires 
n'ont pas leur réplique dans le monde astral. Voici enfin une histoire de marin propre à démontrer que, différent en cela de ceux de MM. Meinecke et Müller, un fantôme peut se révéler actif et utile. Un marin écossais du nom de Robert Bruce aperçoit dans la cabine du capitaine de son navire, occupé à écrire, un étranger dont il ne reconnaît pas les traits. Prévenu, le capitaine découvre un message laissé par l'étranger : «barre au nord-ouest ». L'homme a disparu, mais on suit ses instructions, et l'on sauve l'équipage d'un navire en perdition. Or le capitaine de ce bâtiment avait les mêmes traits et la même écriture que le fantôme (Die monistische Seelenlehre, 243-244).

Les tâches imposées aux doubles sont parfois pénibles. Max Dessoir résume ainsi les pérégrinations imposées au corps astral d'un médium de Cahagnet :

Ganz wundersam sind Adèles Erlebnisse. Sie sandte ihre Psyche nach Mexiko, sah dort einen Lebenden, nach dem gefragt worden war, berichtete, was er trieb und wie schwer es sei, ihn zum Reden zu bringen. Bei dieser Sitzung hielt sich Adèle die linke Hand vor die linke Gesichtshälfle, konnte aber nicht verhindern, daß diese von der mexikanischen Hitze blaurot wurde; die Färbung verschwand erst nach 24 Studen. Sonderbar, höchst sonderbar! Der Astralleib spaziert in Mexiko herum, schwitzt und übertragt nach seiner Heimkehr die Hautentzündung auf den Zellenleib. So muß es doch wohl gewesen sein?

(Dessoir, op. cit., 173)

C'est à la suite d'une expérience personnelle qu'il a notée surie-champ avant de la rapporter dans Das Truggebilde, que Menninger-Lerchenthal entreprit ses recherches :

Stenogramm: «Beim Einschlafen ist mir auf einmal gegenwärtig (optisch, aber nicht lebhaft, nicht visionär), wie ich aus üblicher bestimmter Richtung auf das Haus zugehe, um mich in mein Zimmer zu begeben. Dabei sehe ich halb rechts zum ersten Stock hinauf und dort aus dem Fenster meines Zimmers einen etwa fünfzig Jahre alten Herrn mit ruhigem, ausdruckslosem "Vollmondgesicht" herunterschauen, den ich nicht kenne und trotz Nachdenkens im Halbschlaf und dann eingeschaltetem Wachzustande nicht erkenne, mit dem ich mich aber gefühlsmäßig teilweise identifiziert vorkomme. Es kommt mir so halb und halb vor, als ob der Mann, den ich herunterschauen sehe, ich selbst wäre». (10-11).

On aura relevé les points suivants : 1) que l'expérience se produit lorsque le sujet est dans un état de demi somnolence ; 2) qu'il s'identifie progressivement, et de manière imparfaite, à la vision ; 3) que, Join d'être une image mentale, cette dernière présente un caractère "esthésique» indéniable, encore que relativement faible; 4) que l'impression d'identité continue à s'imposer, malgré le réveil de l'esprit critique ;5) que cette impression d'identité a une certaine coloration affective. L'auteur, qui a l'impression de se voir hors de lui-même, ne fait donc de la vision ni une réalité autonome, ni une illusion banale, ni une simple image mentale. Alors que, pour un occultiste, c'est à son caractère esthésique qu'on reconnaît un « corps astral » échappé de son enveloppe charnelle, pour le psychologue, c'est avant tout l'état affectif du sujet qui impose l'impression d'identité.

Dans son second ouvrage, le même auteur décrit avec précision l'expérience telle qu'elle s'offre sous son aspect le plus saisissant :

Die Truggestalt befindet sich wie eine wirkliche im Raume, in der nächsten Umgebung des Heautoskopen. Sie schwebt nicht in der Luft, sondern steht fest auf dem Boden, geht oder sitzt auf einem Sessel. Oft hören wir, daß die Gestalt sich 
unmittelbar gegenüber befunden habe. Eben so oft wird aber auch die Entfernung von der wirklichen Person zur Truggestalt genau in Metern angegeben. Die Erscheinung tritt, was jetzt nicht mehr nachgewiesen werden muß, so leibhaftig vor Augen, daß, wer sie hat, heftig erschrickt, manchmal sogar einen Schrei des Entsetzens ausstößt. Verliert der Mensch nicht die Besonnenheit, was z. B. dann der Fall ist, wenn er diese Erscheinung schon kennt, dann tritt er auf sie zu, will ihr die Hand reichen oder ihr auf die Schultern klopfen. In diesem Augenblicke entschwindet die Gestalt, die überhaupt selten länger als einige Augenblicke zu sehen ist.

Das zweite Hauptmerkmal des echten Truggebildes der eigenen Gestalt, das wir hier vornehrnlich im Auge haben, sind Empfindungen, die die Heautoskopen dabei haben. Sie sagen, sie hätten das Gefühl gehabt, aus sich herausgetreten zu sein; oder etwas sei aus ihrern Körper ausgetreten, die Seele sei entwichen, dit leere Körperhülle sei zurückgeblieben. Das sind nicht etwa bloße Redensarten, die immer wierderkehren, sondern es sind die Worte, mit denen allein das, was während der Selbstschau empfunden wird, zum Ausdruck gebracht werden kann. [...] Diese Empfindung während des Erlebnisses der Heautoskopie ist zumindest so eindrucksvoll wie die leibhaftigste Gesichtssinnestäuschung. Sie dürfte hauptsächlich der Heautoskopie den Charakter des Erlebnisses geben: denn wohl an ihr liegt es, daß der Heautoskope zur Überzeugung gelangt, daß mit ihm wirklich etwas vorgehe und nicht vielleicht nur eine Tauschung vorliege.

(Der eigene Doppelgänger, 8-9)

À comparer cette description à l'observation personnelle de Menninger-Lerchenthal, on est amené à conclure que l'expérience héautoscopique comporte différents degrés. À en croire Axel Munthe, Mau passant l'aurait éprouvée sous sa forme la plus aiguë :

often he used to stand for minutes staring at himself in the mirror over the mantelpiece as if he was looking at a stranger. One day he told me that while he was sitting at bis writing-table hard at work on his new novel he had been greatly surprised to see a stranger enter his study notwithstanding the severe vigilance of his valet. The stranger had sat down opposite him at the writing-table and began to dictate to him what he was about to write. He was just going to ring for François to have him tumed out when he saw to his horror that the stranger was himself.

(The Story of San Michele, chap. XVIII : « La Salpêtrière »)

Or, il faut compléter la phénoménologie, c'est-à-dire la description de l'« Erlebnis », par la recherche de ses causes. Nous serons en droit de penser que la vision du double n'est qu'une illusion si nous sommes en mesure de lui assigner une origine psychophysiologique, et surtout si nous sommes capables de la susciter expérimentalement à loisir. Mais c'est une tâche que nous ne pourrons mener à bien que partiellement, pour cette raison que l'expérience héautoscopique ne revêt ses formes les plus nettes que chez les malades atteints d'infections organiques graves.

Il est cependant permis de penser que la vision du double n'est autre chose qu'un trouble de la somatognosie, «eine Störung des Körperlichkeitsempfindens». Si l'anatomie nous renseigne sur l'emplacement de nos organes, peu de gens ont pratiqué cette discipline. Cependant, tout homme a de la situation de la plupart de ses organes, externes surtout, une connaissance innée qui va se perfectionnant pendant l'enfance. Si je vous demande de fermer les yeux et de poser votre index sur l'extrémité de votre nez, sur votre genou gauche ou sur votre gros orteil droit, vous exécuterez les gestes demandés sans erreur ni hésitation, et sans vous demander quel guide a dirigé un organe aveugle dans l'obscurité. Or, il setrouve que tel malade cherche son nez à une place où il ne se trouve pas, ou que tel autre, ne sachant plus où sont ses mains, estime qu'on les lui a coupées. Et, bien entendu, beaucoup de mutilés continuent d'éprouver la 
présence d'un membre amputé, d'y ressentir des douleurs, et de croire pouvoir s'appuyer sur lui sans se douter qu'ils s'exposent à une chute. N'en induisez pas que leur corps astral s'est détaché de leur corps physique, mais simplement que leur somatognosie est altérée. L'expérience héautoscopique est parfois le symptôme d'une affection organique grave, comme l'épilepsie, ou d'une lésion de l'hémisphère cérébral droit. Elle accompagne fréquemment des troubles vestibulaires, comme en témoigne l'impression singulière que chacun peut ressentir lorsque s'arrête brutalement un ascenseur : «beim Stoppen des hinunterfahrenden Lifts; unter den wirklichen Füßen empfinden [wir] zwei leichtere Phantomfüfie» (15). Cette impression tient à une «Dissoziation des Körpererlebens unter dem Einfluß von Vertikalbeschleunigungen. Eine solche Dissoziation im Körpergebilde kann nur mit dem Vestibularapparat in Zusammenhang gebracht werden. Phantomkopf, Phantomfülle, Phantomkörper sind Vestibulartäuschungen» (15).

On objectera vainement que, par des exercices appropriés, les adeptes de certaines sectes parviennent à détacher leur corps astral de leur corps physique. Ils croient en trouver la preuve dans l'impression de vide («Ausgehohltsein») qu'ils arrivent à ressentir. Il est probable en effet que des efforts de concentration mentale, associés ou non à certaines intoxications, produisent cet effet. Mais ils ne font que traduire dans le vocabulaire de leur secte des expériences étranges certes, mais dont l'origine est banale. Cependant, les phénomènes psycho-physiologiques ne sont jamais simples. Sont également dans l'erreur ceux qui estiment que l'état physique dépend exclusivement de l'état mental, et ceux qui soutiennent qu'il en est tout-à-fait indépendant. Or l'expérience héautoscopique est commune aux sujets sains et aux sujets malades. Il existe une série de nuances entre l'impression interne de dédoublement («zwei Seelen leben in meiner Brus!») et l'hallucination héautoscopique. Il semble que la vision du double soit plus fréquente chez les sujets prédisposés à l'eidétisme, à la paramnésie, voire à l'introspection. Les premiers ont la faculté d'apercevoir sur un écran, et de décrire avec un luxe incroyable de détails, une image qu'ils n'ont vue de leurs yeux que quelques instants : ils seraient en mesure de percevoir leur image spéculaire comme n'importe quelle autre. Les seconds, qui ont l'impression, non pas de se ressouvenir d'un moment passé, mais de le revivre, souffrent d'une forme légère de dépersonnalisation à la faveur de laquelle s'estompe le sens du réel. Chez les troisièmes, la vivacité des souvenirs peut être telle, en de certaines circonstances, que les images de la mémoire s'apparentent à celles de la perception; cependant la chose est discutable :

In der Literatur findet man das Sichselbstsehen von der Art der Heautoskopie auch unter die Sinnengedachtniserscheinungen eingereiht; wir glauben aber, daß das nicht genügend berechtigt ist. Es ließe sich darüber diskutieren, wenn man die Sinnengedächtniserscheinungen auf eine abnorm gesteigerte Ansprechbarkeit der optischen Hirnrindenfelder zurückführt, der man in gleicher Weise als Folge krankhafter Prozesse, z. B. bei der beginnenden Schizophrenie, begegnet.

(12)

Cependant, «für die Auffassung der Heautoskopie als eine Sinnengedächtniserscheinung könnten vielleicht die Berichte über willkürliche Heautoskopie angeführt werden». Tel pourrait être le cas de Heine (Buch der Lieder, Heimkehr, XX ; Lyrisches Intermezzo, XXXVIII ; Nachgelesene Gedichte, Lamentationen, XII). 
143 D'autre part, sans prétendre, comme le philosophe Emil Lucka, qu'on n'a jamais rencontré de «Doppelgängerinnen» parce que les femmes sont incapables d'un retour sur soi qui serait le privilège des hommes, E. Menninger-Lerchenthal observe que l'héautoscopie est plus fréquente chez les hommes, et l'hystérie chez les femmes, les premiers étant les plus portés à l'auto-analyse, et les secondes plus suggestibles. Ajoutez à cela les attitudes affectives et mentales imposées par le milieu et le moment. Les gens de lettres d'aujourd'hui dédaignent la pratique de l'introspection en honneur chez leurs devanciers : c'est ce qui expliquerait l'intérêt que portèrent au motif du double les écrivains masculins du siècle dernier ${ }^{15}$.

\section{VII}

Les histoires de diables, de sorciers et de loups-garous ont alimenté en «Gruseln» le vulgaire comme le docte jusqu'à la fin du XVII ${ }^{\mathrm{e}}$ siècle. Puis, revenants et illuminés se partagèrent la faveur du public. Le magnétisme fit son apparition, entraînant dans son sillage le spiritisme : cessant de craindre le retour des morts, petits-bourgeois déçus par la religion officielle et savants vieillissants touchés par la grâce spirite souhaitèrent s'entretenir avec les défunts. L'attrait ambivalent du merveilleux, mélange de curiosité et de crainte, incita à faire tourner les tables et consulter les voyantes. D'autres pratiquèrent ce que Coleridge appelait une suspension volontaire de l'incrédulité : ils prirent goût à une littérature fantastique qui leur fournit en abondance des récits invraisemblables et donnés pour fictifs. Puis cette nourriture parut fade: on l'assaisonna des piments de l'érotisme, on proposa aux palais blasés les ragoûts faisandés de l'horreur. Les psychanalystes s'intéressèrent au merveilleux, le plus souvent pour le dépouiller de son charme. Freud écrit par exemple dans sa Traumdeutung:

Die Räuber, nächtlichen Einbrecher und Gespenster, vor denen man sich vor dem Zubettgehen fürchtet, und die auch gelegentlich den Schlafenden heimsuchen, entstammen einer und derselben infantilen Reminiszenz. Es sind die nächtlichen Besucher, die das Kind aus dem Schlafe geweckt haben, um es auf den Topf zu setzen, damit es das Bett nicht nässe, oder die die Decke gehoben haben, um sorgsam nachzuschauen wie es wahrend des Schlafens die Hände hält. Aus den Analysen einiger dieser Angstträume habe ich noch die Person des nächtlichen Besuchers zur Agnoszierung bringen können. Der Räuber war jedesmal der Vater, die Gespenster werden wohl eher weiblichen Personen im weißen Nachtgewande entsprechen.

$$
\text { (VI. «Die Traumarbeit», § E) }
$$

145 Ces lignes ne sont pas les meilleures d'un auteur qui, dans Totem et Tabou, exposa des idées intéressantes, encore que discutables. Qu'on me permette de leur préférer ce passage de Walter F. Otto :

Wir haben in den «Totenerscheinungen» ein Phänomen kennengelernt, das weder von krankhafter Veranlagung noch von einer Glaubenstradition abhängig ist und das in allen Ländern bei Menschen jedes Bildungsgrades und zu allen Zeiten in der gleichen Weise auftritt. Der Totenglaube, dessen älteste und dauerhafteste Gestalt wir erkannt haben, entspricht diesem Phänomen Zug um Zug. Keine Hypothese vermag ihn unserem rationalen Denken begreiflich zu machen. Wir müssen ihn zurückführen auf einen uns Heutigen oder wenigstens unserer wissenschaftlichen Betrachtungsweise fremdgewordenen Eindruck und Vorstellungsprozeß, die zu ergründen der Psychologie vorbehalten bleibt. 
(Die Manen, oder Von den Urformen des Totenglaubens, Sonderausgabe, Darmstadt, 1983, S. 114$)$. part de leurs visions et de leurs prémonitions, a chargé sa collaboratrice Aniela Jaffé d'analyser les réponses. Faute de contrôle, celles-ci ne pouvaient retenir l'attention des parapsychologues; faute d'originalité, elles ne pouvaient enrichir la littérature fantastique. Elles se donnaient cependant pour des relations d'expériences vécues authentiques. Mais à quel signe les reconnaître comme telles? À première vue, le critère retenu paraît entaché du vice de circularité : les expériences authentiques sont celles qui confirment les théories de Jung, et réciproquement. Or Jung répond dans la préface du livre : «Es besteht [...] kein zureichender Grund, an der Wahrhaftigkeit eines einzelnen Berichtes prinzipiell zu zweifeln. Ein berechtigter Zweifel ist nur dort angebracht wo es sich um eine absichtliche Lüge handelt. Die Anzahl solcher Fälle ist verschwindend klein, denn die Urheber solcher Falschungen sind zu unwissend, um richtig lügen zu kännen» (Geistererscheinungen und Vorzeichen, Zürich, 1958, 11). L'auteur en donne un exemple convaincant (Ibid., 263-264). Elle reproduit les récits tels quels. «Dadurch [schreibt Jung] bleibt die dem Bericht wesentliche Atmosphäre, das Zwielicht, erhalten. Zum nachtlich'numinosen Erlebnis gehärt die Bewußtseinsdammerung, das Ergriffensein, die Unmäglich keit der Kritik und die Lähmung der eigenen Stellungnahme» (11). C'est surtout la signification des expériences vécues, de la perception des visages enveloppés de lumière, des dames blanches, des fantômes sans têtes, des esprits des morts qui ont revêtu l'apparence des vivants, qui intérèsse Aniela Jaffé. Si hasardeuse qu'elle soit, cette psychologie s'efforce de décrire le vaste domaine qui sépare la réalité objective de l'imagination gratuite.

De leur côté, les théologiens paraissent avoir renoncé à spéculer sur la persistance des fonctions sensorielles et digestives des corps glorieux, pour pratiquer une «Verstummung vor dem "Mysterium"». À vous permis de décider si ce renoncement à la «cataphase » en faveur de l'« apophase » est l'indice d'une perception plus aiguë du mystère, ou le prétexte d'une dérobade devant les questions précises (voir : G. Greshake u. J. Kremer, Resurrectio Mortuorum. Zum theologischen Verständnis der leiblichen Auferstehung, Darmstadt, 1986).

Je citerai pour conclure les opinions de trois philosophes allemands au sujet de la survie personnelle. La première, qui ressort d'une confidence de Kant à Jachmann, ne contredit pas, en dépit des apparences, la croyance du philosophe à l'immortalité de l'âme considérée comme postulat de la raison pratique :

Wir kamen eines Tages in einern vertrauten Gespräche auf diesen Gegenstand, und Kant legte mir die Frage vor: was ein vernünftiger Mensch mit voller Besonnenheit und reifer Überlegung wohl wählen sollte, wenn ihm vor seinem Lebensende ein Engel vom Himmel, mit aller Macht über sein künftiges Schicksal ausgerüstet, erschiene und ihm die unwiderrufliche Wahl vorlegte und es in seinen Willen 
stellte, ob er eine Ewigkeit hindurch existieren oder mit seinem Lebensende gänzlich aufhören wolle? und er war der Meinung, daß es hächst gewagt wäre, sich für einen völlig unbekannten und doch ewig dauernden Zustand zu entscheiden und sich willkürlich einem ungewissen Schicksal zu übergeben, das ungeachtet aller Reue über die getroffene Wahl, ungeachtet alles Überdrusses über das endlose Einerlei und ungeachtet aller Sehnsucht nach einem Wechsel dennoch unabänderlich und ewig wäre.

$$
\text { (Jachmann, op. cit., Elfter Briel) }
$$

La seconde est celle que hasarde Richard Baerwald (1867-1929) à la fin de son livre : okkultismus und Spiritismus und ihre weltanschaulichen Folgerungen (Berlin, 1926). Comparant notre existence terrestre à la vie fade et aux occupations puériles des « esprits » dans l'au-delà, il en vient à se demander si, en dépit de toutes ses horreurs, notre univers n'est pas, comme le soutenait Leibniz, le meilleur des mondes possibles.

La troisième est de Max Dessoir (1867-1947) achevant, quelques mois avant la fin d'une vie consacrée à la para psychologie, son dernier livre : Das Ich, der Traum, der Tod:

Ich bin kein grimmiger Aufklärer, für den «Geister nur irrtümliche Bettlaken» sind, sondern ich halte es mit Sir Archie in Hauptmanns Winterballade: «Die Welt ist voller Magie». Freilich nicht voller Totengeister.

\section{NOTES}

1. La correspondance de Spinoza et de Hugo Boxel est le prétexte d'un récent « Essai sur l'esprit philosophique » d'Alain BILLECOQ : Spinoza et les spectres, Paris, P.U.F., 1987.

2. Exposé dense et précis dans E.R. DODDS, «The Astral Body in Neoplatonism» (Proclus, The Elements of Theology, 2nd ed., Oxford, 1962, p. 313-321). Consulter: J. TROUILLARD, L'Un et l'Ame selon Proclus, Paris, Les Belles Lettres, 1972, p. 184-189. E.A. MOUTSOPOULOS, Les Structures de l'imaginaire dans la philosophie de Proclus, Paris, Les Belles Lettres, 1985, et, panni les auteurs moins récents : R. CUDWORTH, The True Intellectual System..., London, 1678, p. 767-842, Carl du PREL, Die Monistische Seelenlehre, Leipzig, E. Günther, 1888.

3. J. GLANVILL, «The True Notion of a Spirit», in Saducismus Triumphatus..., London, 1681.

4. Rédigée dans un allemand quasi inintelligible pour un lecteur moderne, l'œuvre de Paracelse fut «traduite» au XIXe siècle. W.-E. PEUCKERT a su trouver un compromis heureux en présentant un texte lisible qui conserve autant que possible la saveur de l'original (Theophrastus Paracelsus. Werke, Darmstadt, WissenschaftHche Buchgesellschaft, 1965-1976). Les 4 premiers volun1es reproduisent l'essentiel de l'œuvre authentique, le $5 \mathrm{e}$ comprend des écrits généralement tenus pour apocryphes, mais qui ont, plus que les autres peut-être, servi la réputation de leur auteur supposé. - On regrettera que cette édition ne comporte ni notes, ni index, ni tables de matières détaillées. C'est elle que je cite.

5. D’après les notes de Robert BLUM incorporées dans la 2e édition de la Geschichte des neueren Occultismus de Karl KIESEWETTER (Leipzig, M. Altmann, 1900).

6. La page est digne de Rabelais : «Ad illud, an visibiliter vel inuisibiliter huiusmodi spurcitias agant mutuò, dicendum quantum experientia nos edocuit, quòd licet semper visibiliter ex parte maleficae Daemon Incubus operetur, ita quòd non est necessarium inuisibilîter propter foederatum pactum expressum ei appropinquare. Tametsi quoad circunstantes, saepius ipsae maleficae supinae iacentes in agris, seu syluis visac sunt, \& denudatae supra vmbilicum, \& iuxta 
dispositionem illius spurcitiae coaptatis membris, tibiis \& cruribus se agitantes. Daemonibus Incubis, inuisibiliter ibidem quoad circumstantes cooperantibus: licet in fine actus, vapor nigerrimus in longitudine hominis sursum à malefica in aëre eleuabitur, sed hoc rarissimè» (Malleus maleficarum, Lugduni, 1669, II, Q. I Cap IV, p. 122 A-B).

7. D'après Ernest BORNEMANN, l'expression «amor hercos» aurait été forgée au début du XIVe siècle par un médecin de Montpellier, Bernard de Gordon, comme synonyme de l'amour considéré comme une maladie mentale (Das große Wörterbuch der Sexualität, München, M. Pawlak, $1984, \mathrm{~s} . \mathrm{v}^{\circ}$ ). Il ne semble pas toutefois que cette définition corresponde à l'usage qu'en fait Paracelse, et qu'il faille en chercher le sens à une époque antérieure ; «Haereo has a wide range of attested sexual uses: of copulation at Prop. 2.15.25. "atque utinam haerentis sic nos uincire catena / uelles", Petron 79.8. "haesitnus calentes" (cf. Lucr. 4.113, 1205, and cohaereo at Plin. Nat, 10. 173), of manual stimulation at Juv. 6.024 "saepius in teneris haerebit dextera lumbis", and of cunnilinctio at Mart. 11.61.11 "tumenti mersus haeret in uolua". Cf. Mari. 2.61.7 "haereat inguinibus potius tam noxia lingua", Sen. N.Q. 1.16.4. "inguinibusque alienis obhaeserat" Min. Fel. Oct. 28. 10. "libidinoso ore inguinibus inhaerescunt", where the complements leave no doubt concerning the type of "clinging" in question » (J .N. AoAM, The Latin Sexual Vocabulary, London, Duck worth, 1982, p. 181-182).

8. Dans un ouvrage intitulé Traité par dialogue de l'énergie ou opération des diables, Michel Psellos, érudit byzantin du XIe siècle, rapporte les propos qu'un certain Capitaine de Thrace tient d'un hérétique converti, frère Marc, et communique à son interlocuteur Timothée: «Et de fait, m'étant quelquefois enquis de lui, s'il y a quelques diables patibles : Oui vraiment, dit-il, comme on dit aussi qu'aucun d'iceux jettent semence et engendrent d'icelle des verms [vers] - Si est-ce chose incroyable, lui dis-je lors, que les diables aient aucuns excréments, ni membres spermatiques, ni vitaux. - Vrai est, répondit-il, qu'ils n'ont tels membres ; si est-ce toutefois qu'ils jettent hors je ne sais quel excrément et superfluité». Une traduction française du ПЕPI ENEPTEIA $\triangle A I M O N \Omega N$ de Michel PSELLOS [par Pierre Moreau], Revue des Etudes grecques, $t$. XXXIII. 1920, p. 74.

9. Le passage des Vorlesungen über die Metaphysik de Kant est cité d'après l'édition originale de Pülitz, Erfurt, 1821. Pülitz lui-même avait fait paraître une étude intitulée : Können höhere Wesen auf den Menschen wirken und sich mit ihm verbinden?, Leipzig, 1794. Les passages de la lettre à Charlotte von Knobloch sont empruntés à la Durstellung des Lebens und Charakters Immanuel Kants de L.E. BOROWSKI, reproduite dans : Immanuel Kant. Sein Leben in Darstellungen von Zeitgenossen hrsg. von Felix Groß, Berlin, 1912, S. 96-103. Les autres textes de Kant sont empruntés à : Kants gesammelte Schriften hrsg. von der Küniglich Preußischen Akademie der Wissenschaften, Bd II.

10. Johann Heinrich JUNG, dit STILLING est surtout connu par son attachante autobiographie (Lebensgeschichte). Je cite sa Theorie der Geister-Kunde, in einer NaturVernunft- und Bibelmäsigen Beantwortung der Frage : was von Ahnungen, Gesichten und Geistererscheinungen geglaubt und nicht geglaubt werden müße d'après l'édition originale, Nürnberg, 1808. L'ouvrage comprend 255 paragraphes répartis en 4 «Hauptstücke ». Le cinquième " Hauptstück », intitulé ; Kurze Uebersicht meiner Theorie der Geisterkunde, und Folgerungen aus derselben compte 55 paragraphes numérotés de 1 à 55 .

11. Les Parapsychologische Schriften de SCHOPENHAUER ont été réunis, précédés d'une intéressante introduction de Hans Bender, en un vol ume qui regroupe les textes suivants: Animalischer Magnetismus und Magie, Transzendentale Spekulation über die anscheinende Absichtlichkeit itn Schicksale der Einzelnen, Versuch über das Geistersehn und was damit zusammenhängt, Zur Lehre der Unzerstörbarkeit unsers wahren Wesens durch den Tod. C'est cette édition que je cite.

12. Justinus KERNER, Die Seherin von Prevorst, II. Teil (Werke hrsg. von Raimund Pissin, Berlin, 1914, 5. Teil, S. 133-134).

13. Justinus KERNER, Die Seherin von Prevorst, I. Teil (Werke, 4. Teil, S. 224). 
14. Il lui épargne cependant les compliments qu'il réserve à la philosophie de Hegel: «Mystifikation Farce», «Narrenpossen», «Galimathias», «Wischiwaschi», «Afterweisheit». 15. - Sur les aspects littéraires du problème, consulter outre le livre classique d'Otto RANK, Don Juan et le Double, trad. S. Lautman, Paris, Denoël, 1932, et «Petite Bibliothèque Payot ", vol. 211, l'article d'Elisabeth FRENZEL : «Doppelgänger» dans ses Motive der Weltliteratur, Stuttgart, A. Krüner, 1976. On trouvera d'intéressantes observations dans le livre de Celia GREEN, Out-of-theBody Experiences, Oxford, Institut for Psychophysical Research, 1968, et l'excellent Lexikon der Parapsychologie de F. BONIN, Herrsching, M. Pawlak, 1984. Parmi les ouvrages psychiatriques français publiés après ceux de Menninger-Lerchenthal, on retiendra surtout celui de Jean LHERMIITE, Les Hallucinations. Clinique et Physiopathologie, Paris, O. Doin, 1951 (en particulier p. 124-168) et celui de H. HECAEN et J. de AJURIAGUERRA, Méconnaissances et Hallucinations corporelles. Intégration et Désintégration de la Somatognosie, Paris, Masson et Cie, 1952 (surtout p. 318-370). L'étude philosophique assez partiale d'Emil LUCKA, «Verdoppelung des Ich» a paru dans les Preußische Jahrbücher, CXV. Bd (1904), S. 54-83.

Remarques: 1) Les citations de cet article respectent, sans souci de modernisation ou d'uniformité, l'orthographe des ouvrages auxquels elles sont empruntées. 2) Daignent les lecteurs vétilleux me pardonner l'usage des termes d'occultiste et d'occultisme, anachroniques certes, mais utiles.

\section{RÉSUMÉS}

Depuis toujours, les fantômes et les entités apparentées, doubles, corps astraux, esprits qui hantent les ténèbres etc. ont attiré l'attention des philosophes. Parmi les auteurs de langue allemande, Paracelse soutient que l'homme est pourvu de deux corps, l'un charnel, l'autre subtil. La mort les voue l'un et l'autre à la décomposition : le premier dans la terre, et le second, qui n'est pas un esprit, mais une chose morte, dans l'atmosphère.

Par ailleurs, c'est de l'imagination des luxurieux que naissent incubes et succubes. Bien que personnellement enclin à nier l'existence des «esprits » Kant estime qu'il est permis d'en admettre la possibilité sans risquer d'être contredit. De son côté, Jung-Stilling décrit en long et en large le royaume des esprits. Schopenhauer défend quant à lui une théorie métaphysicophysiologique selon laquelle le magnétisme, les rêves lucides, la seconde vue et les apparitions, faits avérés, prouvent l'existence d'une «Volonté » indépendante de l'espace, du temps et de la causalité. Du Prel considère que la perception du double est celle d'un corps éthéré qui s'est séparé du corps physique. En revanche, MenningerLerchenthal juge illusoire cette prétendue séparation: la vision du double n'est à son sens qu'un symptôme de quelque trouble de la somatognosie. - En conclusion, l'auteur partage cette opinion de Max Dessoir, selon qui le monde est plein de magie, mais qu'il n'est assurément pas hanté par les fantômes des morts.

Von jeher haben die Gespenster und die mit ihnen verwandten Wesen (Doppelgänger, Astralkörper, Nachtgeister u.a.m.) die Aufmerksamkeit der Philosophen auf sich gezogen. Unter den deutschsprachigen Autoren behauptet Paracelsus, daß der Mensch mit zwei Körpern : einem fleischlichen und einem feinstofflichen versehen ist. Nach dem Tode verwesen beide: der eine in der Erde, der andere, der kein Geist sondern ein totes Ding ist, in der Luft. Anderseits ist die Imagination der Unkeuschen der Ursprung der incubi und succubi. Über das Dasein der «Geister» neigt Kant zur verneinenden Seite. Man kaon aber seines Erachtens die Möglichkeit solcher 
Wesen ohne widerlegt zu werden annehmen. Seinerseits beschreibt Jung-Stilling das Geisterreich ausführlich. Seiner metaphysisch-physiologischen Theorie gemäß behauptet Schopenhauer, daß Magnetismus, Wahrtrtäume, zweites Gesicht und Geistersehn Tatsachen sind, welche die Wirklichkeit eines von Raum, Zeit und Kausalität unabhtängigen «Willen» beweisen. Laut du Prel ist die Doppelgängerei eine Manifestation des abgetrennten Ätherleibes. Dagegen versichert Menninger-Lerchenthal, daß diese vermeintliche Trennung eine Illusion ist: die Doppelgängererscheinungen sind nur Symptome einer Störung des Körperlichkeitsempfindens. Zum Abschluß stimmt der Verfasser mit dieser Überzeugung Max Dessoirs überein: «Die Welt ist voller Magie, freilich nicht voller Totengeister».

\section{AUTEUR}

\section{LOUIS VAX}

Université de Nancy II 